\title{
The Composition of Volcanic Ash and the Dynamics of the 2013-2016 Zhupanovsky Volcano Eruption
}

\author{
N. V. Gorbach ${ }^{a}$ *, A. A. Plechova ${ }^{b}$, T. M. Manevich ${ }^{a}$, \\ M. V. Portnyagin ${ }^{c}$, T. M. Philosofova ${ }^{a}$, and S. B. Samoilenko ${ }^{a}$ \\ ${ }^{a}$ Institute of Volcanology and Seismology, Far East Branch, \\ Russian Academy of Sciences, bul'var Piipa, 9, Petropavlovsk-Kamchatskii, 683006 Russia \\ ${ }^{b}$ Vernadsky Institute of Geochemistry and Analytical Chemistry, \\ Russian Academy of Sciences, ul. Kosygina, 19, Moscow, 119334 Russia \\ ${ }^{c}$ Helmholtz Centre for Ocean Research Kiel, GEOMAR, Kiel, Germany \\ *e-mail:n_gorbach@mail.ru \\ Received March 17, 2017
}

\begin{abstract}
This paper presents the results from a study of ash compositions that were erupted in 2013-2016. The juvenile component has been identified in the ejecta using data on the morphology and textural features of ash particles and the composition of volcanic glasses. The data set suggests that the activity of the volcano was phreatomagmatic.
\end{abstract}

DOI: $10.1134 / \mathrm{S} 0742046318030028$

\section{INTRODUCTION}

The study of volcanicash compositions is an effective monitoring tool for the observation and prediction of eruptive activity along with seismic observations and remote sensing data (Ponomareva et al., 2012; Suzuki et al., 2013; Cashman and Hoblitt, 2004; Taddeucci et al., 2002, among others). The method acquires special significance if an eruption or its initial phase is phreatic or phreatomagmatic in nature. In this case, tephra is one of the few available sources of data on the processes in the magmatic system of the volcano, while the key problem is to determine the presence, amount, and composition of juvenile material in the ejecta. One of the most successful examples of such research is the survey of Unzen Volcano in Japan, when a study of a series of ash samples from the 1991 eruption succeeded in detecting the appearance of juvenile material three months before the extrusive dome began to grow (Watanabe et al., 1999; Nakada et al., 1995). A petrologic study of ash samples from the eruption of Eyjafjallajoekull Volcano, Iceland along with data of seismic monitoring allowed estimation of the rate of magma ascent and of the duration of magma mixing (Portnyagin et al., 2011; Sigmarsson et al., 2011). Ponomareva et al. (2012) were the first to demonstrate how the current state of the magmatic system of an active volcano in Kamchatka could be estimated based on studies of volcanic glass compositions in the tephra of recent eruptions.

The present paper presents results from a study of ash compositions in the ejecta of Zhupanovsky Vol- cano from 2013 to 2016. We present data on the chemical and component composition of ash ejected by major explosive eruptions. We sought to identify the juvenile component by studying the morphology and textural features in ash particles and the composition of volcanic glasses that we sampled from the ash and products resulting from the July 2015 collapse of the active cone. This data set was used to infer that the eruption was phreatomagmatic in character. It is shown that the collapse of a sector of the active cone might have influenced the dynamics of the subsequent explosive events.

\section{THE ACTIVITY OF THE VOLCANO IN 2013-2016 A Description of the Object of Study}

Zhupanovsky Volcano is situated $70 \mathrm{~km}$ north of the town of Petropavlovsk-Kamchatskii (Fig. 1a) and is part of the Dzenzur-Zhupanovsky volcanic cluster in the East Volcanic Belt, Kamchatka.

The Dzenzur-Zhupanovsky cluster is a complex volcanic massif that includes, in addition to the four cones of Zhupanovsky Volcano, the heavily eroded edifices of Dzenzur, Yur'evskii, Sirenevyi, and Tetyaev, and the related extensive lava fields (see Fig. 1b). The Zhupanovsky cones make up the eastern element in this volcanic cluster. The first and second cones do not possess names of their own, while the third and fourth cones were called Priemysh and Bastion (Bazanova et al., 2009; Litvinov and Burmakov, 

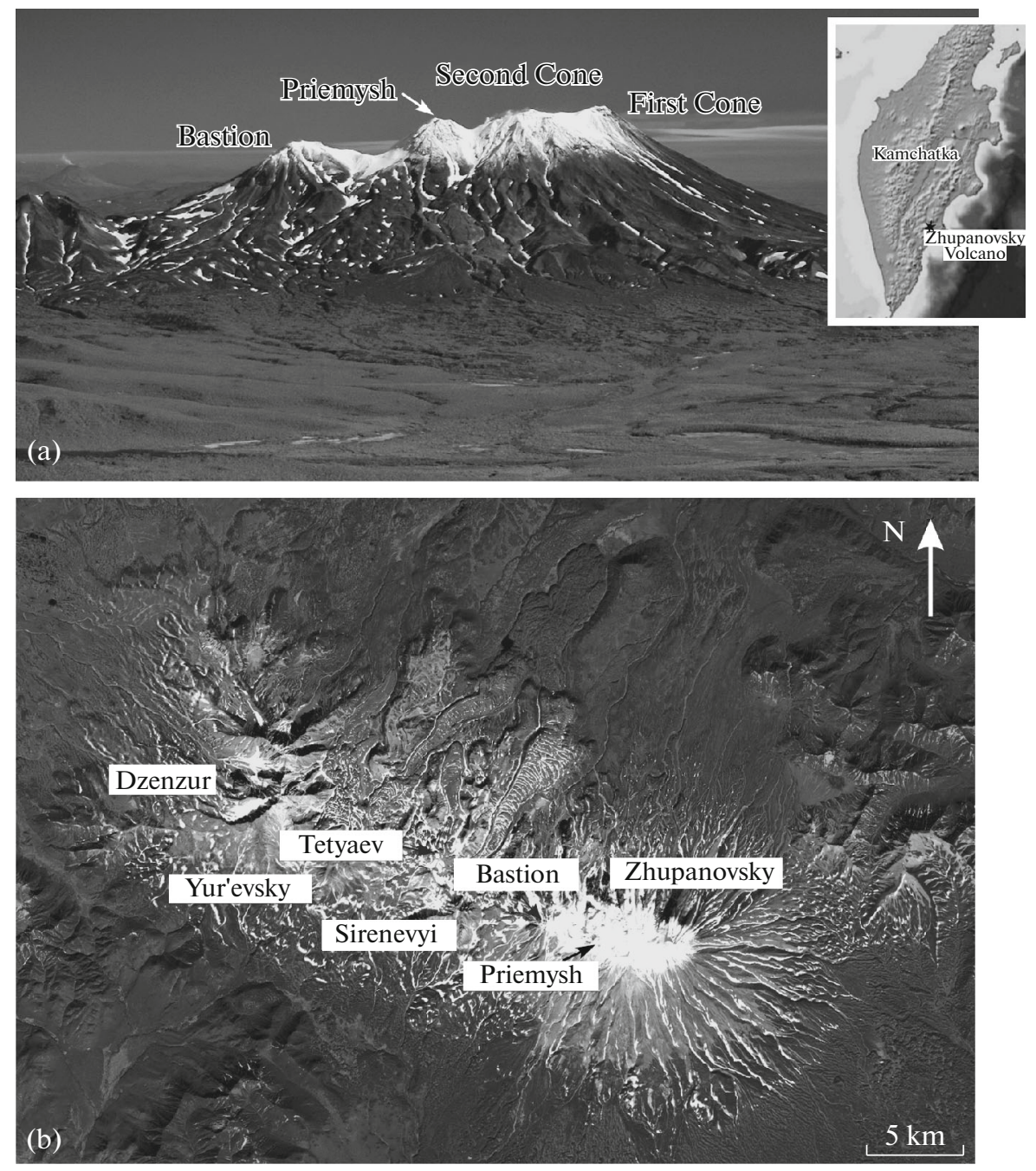

Fig. 1. Zhupanovsky Volcano (a) and its location in the Dzenzur-Zhupanovsky Volcanic Cluster (b).

1993). The first and second cones began to form during Late Pleistocene time. The ejecta of the tallest (altitude $2927.2 \mathrm{~m}$ ) first cone consist of rare porphyritic basalts with an olivine-plagioclase ( \pm pyroxene) paragenesis of the phenocrysts. The second cone is a major pyroclastic lava edifice (altitude $2884 \mathrm{~m}$ ) whose slopes are studded with several cinder cones. The compositions of its lavas varied over time from basaltic andesite and andesite to basalt (Bazanova et al., 2009; Litvinov and Burmakov, 1993; Plechova et al., 2011).

The Priemysh and Bastion cones date from Holocene time and are largely composed of andesitic lavas (Litvinov and Burmakov, 1993; Masurenkov et al., 1991). According to (Puzankov et al., 2016; Bazanova et al., 2009), the Priemysh cone began to be active in the Early Holocene $\left(\sim 7400{ }^{14} \mathrm{C}\right.$ B.P. $)$. The highest level of activity occurred between $\sim 3500-3000$ and $1800{ }^{14} \mathrm{C}$ B.P., while the eruption of $\sim 2100{ }^{14} \mathrm{C}$ B.P. produced pyroclastic pumice flows. Masurenkov et al. (1991) provide information on six weak explosive eruptions of Priemysh during historical time: 1776, 1882, 1925, 1929, 1940, and 1956-1957.

\section{The Dynamics of the 2013-2016 Eruption}

The first series of ash ejections, which rose as high as $5 \mathrm{~km}$ above sea level (a.s.l.) occurred on Priemysh on October 23, 2013 (Samoilenko et al., 2014). The ashfall zone extended southward and southeastward for over $50 \mathrm{~km}$ from the volcano, covering an area of $1200 \mathrm{~km}^{2}$. The ash was $15-20 \mathrm{~cm}$ thick on the cone slopes. The total mass of the ash ejected during the initial phase of the eruption is estimated as 100000 tons.

Steam and gas emission was observed on the volcano during the winter and spring of 2014, while no ash ejections were recorded. Explosive activity was resumed in early June 2014. The ash plume from these explosions extended eastward on June 6-7, with its deposits on snow being traceable as far as the Pacific coast. The ash was $5 \mathrm{~mm}$ thick at a distance of $6 \mathrm{~km}$ 


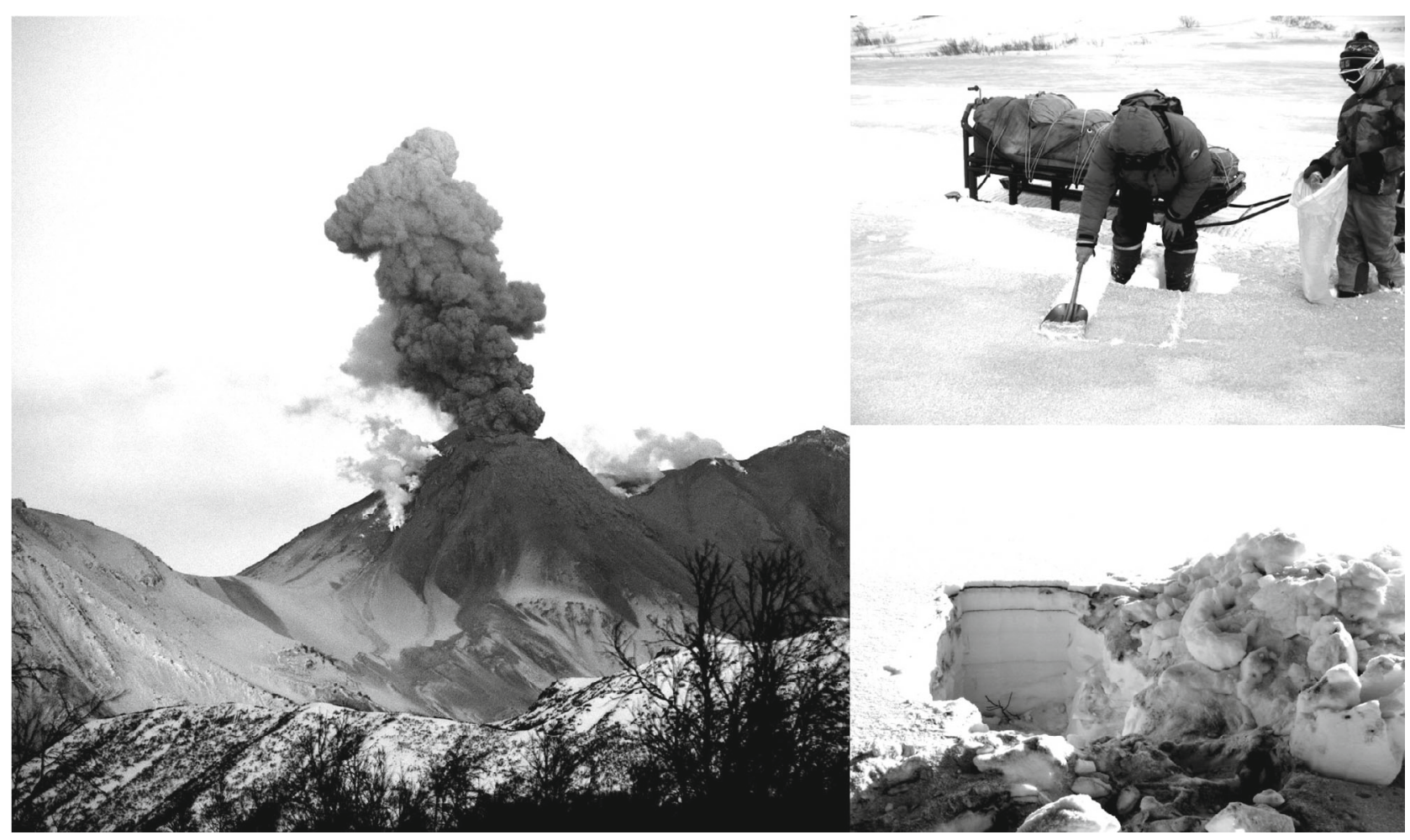

Fig. 2. The explosive activity of Priemysh Cone in January 2015 and ash sampling.

east of the crater, gradually thinning out farther from the volcano, being diminished to a thickness of $1 \mathrm{~mm}$ at a distance of $40 \mathrm{~km}$. The total mass of the ejected ash was 120000 tons. The ashfall covered an area of $1800 \mathrm{~km}^{2}$ (Manevich et al., 2015).

The subsequent 10 months (July 2014 through April 2015) saw episodic ejections of ash from the crater of the active cone (Fig. 2) to heights of mostly 4$5 \mathrm{~km}$ a.s.l. (more rarely as high as $8-10 \mathrm{~km}$ ) with a background steam-gas activity. No explosive activity was recorded during May and June 2015.

A vigorous explosive eruption occurred on July 12, 2015 with accompanying large seismic events in the volcanic edifice. Seismic data suggest that the explosions might reach altitudes of $10 \mathrm{~km}$ a.s.l. during the eruption (Senyukov et al., 2015). The ash plume was the longest to have been recorded during the entire period of activity, with the ash traveling $1100 \mathrm{~km}$ southeast of the volcano (http://www.kscnet.ru/ivs/ kvert/van/index.php? $n=2015-179)$. Another, less powerful, explosive event was observed on July 14. On July 16 it was found that the southern sector of the active cone was destroyed, and the base of the volcano was covered by an extensive field of collapse deposits and mud flows (Gorbach et al., 2015).

Some discrete weak ash ejections occurred between August and October 2015. A series of explosive events followed in late November and early December 2015, with the largest of these (reaching altitudes of 8-9 km a.s.l.) being on November 30. According to (http://www.kscnet.ru/ivs/kvert/van/index.php? $n=$ 2015-211), the event produced pyroclastic or mud flows. A new series of vigorous ash ejections was observed in late January 2016: $8 \mathrm{~km}$ a.s.l. on January 19, and approximately $5 \mathrm{~km}$ a.s.l. on January 21 and 24 . Some discrete powerful ejections were also observed on February 9 and $12(8-10 \mathrm{~km}$ a.s.l.), as well as on March 24, 2016 (Girina et al., 2016). The last explosive event as of the time that this paper was written (December 2016) occurred on November 20, 2016 (http://www.emsd.ru/ ssl/monitoring/main.htm)

\section{Changes in the morphology of the crater zone}

The crater of the Priemysh cone was described in (Litvinov and Burmakov, 1993; Masurenkov et al., 1991; Gorshkov and Slezin, 1972; Sirin, 1958; Piip, 1947). Its diameter was estimated as lying between 200 and $450 \mathrm{~m}$ based on visual determinations. The above descriptions also mentioned a funnel $80 \mathrm{~m}$ across in the crater west of the central vent. Landsat 8-OLI space images (http://landsat.visibleearth.nasa.gov/ view.php?id=82427) show that the crater was no wider than $200 \mathrm{~m}$ before the eruption. The results of airborne visual observations in 2014 and in the earlier half of 2015 along with data from earlier publications indicated that no substantial changes occurred in crater morphology during the initial phases of the eruption. 

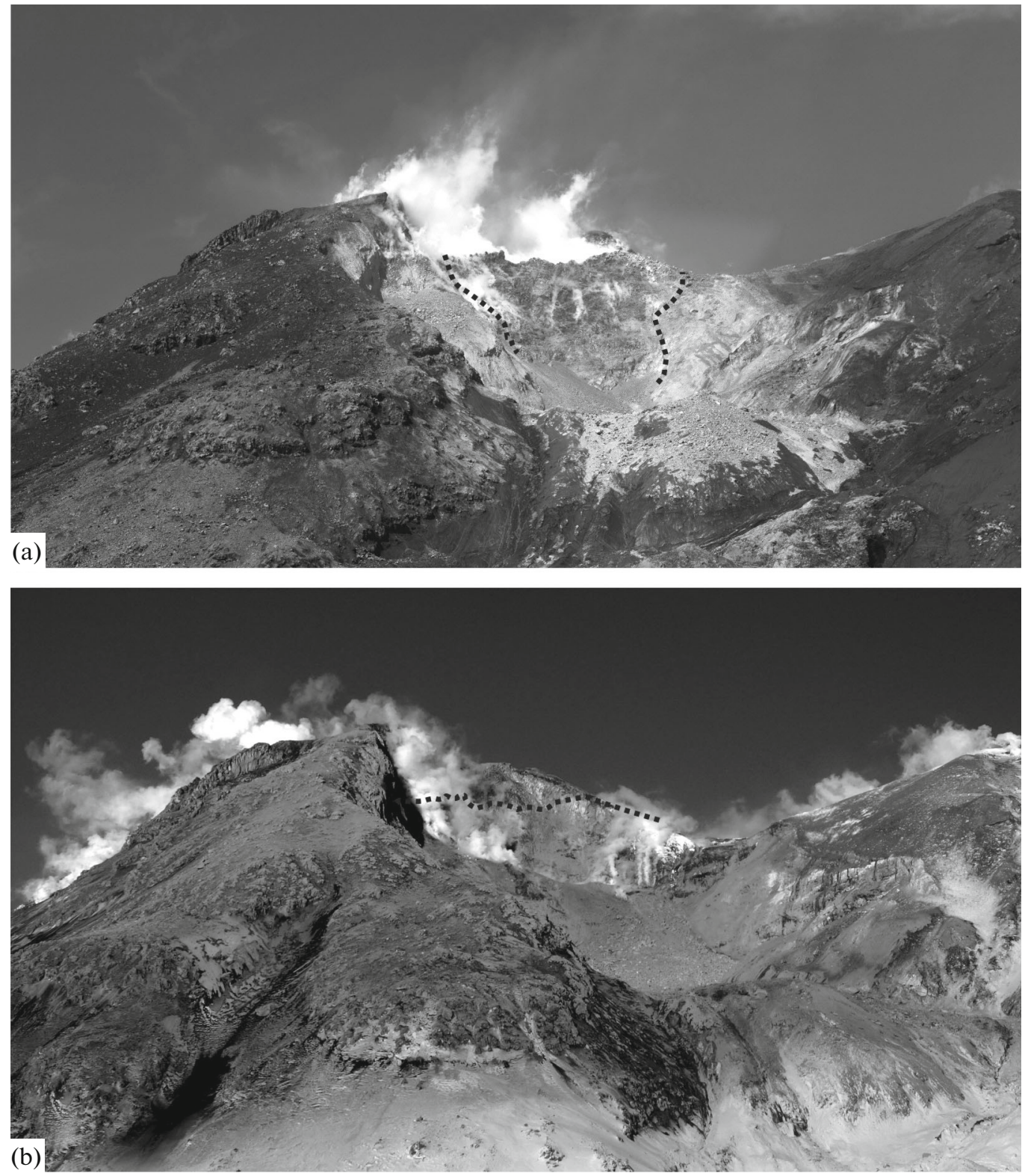

Fig. 3. Changes in the morphology of the crater zone. (a) view from the south of the destroyed sector of Priemysh Cone, July 16 , 2015 , dashes highlight the outline of a neck that was exposed by the collapse; (b) view of the cone from a similar direction, January 23, 2016, dashes mark the upper outline of the neck in July 2015. Photographed by A.A. Plechova (a) and by S.A. Chirkov (b).

The July 2015 explosive activity and the collapse of a sector of the cone produced drastic changes in the appearance of its summit zone. Only the north wall of the crater survived, while its south edge was totally destroyed. The cone slope showed a collapse-explosion cirque opening southward $\sim 500 \mathrm{~m}$ deep and $250-$ $300 \mathrm{~m}$ wide (Gorbach et al., 2015). The walls of this new cirque exposed the structure of the Priemysh summit part and the upper segment of its conduit (Fig. 3a). The explosive activity in the early 2016 somewhat expanded the cirque, altering the profile of the east wall, while the lava body (see Fig. 3b) in its middle was almost entirely destroyed. Remote sensing and field observations did not detect signs of extrusive or effusive activity in the crater, and later in the new collapse-explosion cirque.

\section{SAMPLING AND RESEARCH METHODS}

Ash was sampled during the eruption mostly from snow, most frequently immediately after explosive events. The data on the sampling locations and times are listed in Table 1. Sixteen ash samples from the larger ejections and four samples from the collapse material were used for chemical and mineralogic analyses. The chemical composition of the ashes was 
determined by the X-ray fluorescence method using an S4 PIONEER spectrometer at the Analytical Center of the Institute of Volcanology and Seismology (IV\&S), Far East Branch (FEB), Russian Academy of Sciences (RAS) (Analysts N.Yu. Kurnosova and N.I. Chebrova).

The compositions of volcanic glasses were determined using a JEOL JXA 8200 electron probe at the Helmholtz Centre for Ocean Research Kiel, GEOMAR, Kiel, Germany. A total of approximately 300 determinations were made for the concentrations of major elements, as well as sulfur and chlorine, in glasses from ten representative samples. As well as ash samples, we also analyzed volcanic glass sampled from the cone edifice; this was a crushed (manually) sample of glassy lava sampled from the summit flow and unconsolidated material from the rockfall (Table 2, samples 7650 and 7651-1). The analysis was carried out using a $5-\mu \mathrm{m}$-defocused electron beam with an accelerating voltage of $15 \mathrm{kV}$ and a current of $6 \mathrm{nA}$. The performance of the analysis was tested using basalt, rhyolite, and scapolite standards. A detailed description of the method used for determining the composition of volcanic glass and an account of the data processing procedure can be found in (Ponomareva et al., 2012).

\section{THE MATERIAL COMPOSITION OF THE EJECTA}

\section{The Chemical and Mineral Composition of the Ashes}

A brief description of samples from the larger explosive events and the microscopic aspect of the samples can be found in Table 2. Most samples that were taken at distances of 20 to $50 \mathrm{~km}$ from the eruption center consist of medium-grained and finegrained volcanic sand. The samples from the slopes of the active cone have larger grains. As an example, sample 06-14 ( $4 \mathrm{~km}$ from the eruption center) mostly contains large-grained and medium-grained volcanic sand, while sample 01-16, which was taken at a distance of $\sim 1 \mathrm{~km}$ from the eruption center, contains a small amount of lapilli.

All ash samples are similar in their component composition, mostly consisting of angular dense fragments saturated with microlites, fragments of minerals (pyroxene, plagioclase, and occasionally olivine), and products of hydrothermal activity. The latter include fragments of variously altered rocks, quartz-feldspar aggregates with occasional segregations of sulfides, fragments of gypsum, anhydrites, and other sulfates. The relationships between the ash components are different. The greatest amounts of particles from hydrothermally altered rocks and secondary minerals are found in the ashes ejected by explosive events during the initial phase of the eruption (October 2013, June and October-December 2014), as well as in the ashes ejected on July 12, 2015. The ashes discharged in the early 2015 and in 2016 are dominated by denser fragments (Fig. 4a).

Most of the samples contain small amounts (between $\sim 1$ and 5-7\%) of porous glassy fragments (see Fig. 4b), while the 2016 ejecta were also found to contain pumiceous particles (see Figs. 4c, 4d, and 4e). A few findings were made of fragments of homogeneous glass (see Fig. 4f) and particles that exhibit a combination of patches with different structures (see Figs. $4 \mathrm{~g}$ and $4 \mathrm{~h}$ ). A sample of glassy rock taken from the rockfall field on the cone edifice that was crushed manually is similar to the ash samples in aspect (see Table 2, sample 7651-1) but is less heterogeneous, does not contain hydrothermally altered material, and is dominated by porous particles.

The chemical composition of the ashes ejected by the larger explosive events of 2013-2016 is shown in Table 3. All samples are consistent with moderate potassium andesites (to dacites) of the calc-alkaline series (Fig. 5), which is typical of the Priemysh Late Holocene activity (Bazanova et al., 2009; Puzankov et al., 2016). The silica concentration in the ash samples varies between 56.9 and $65.4 \mathrm{wt} \%$. This wide range of bulk composition resulted from various mixtures of products of hydrothermal activity in the ashes of some individual discharges, which is readily apparent upon comparing Tables 2 and 3. The ashes with the greatest admixture of hydrothermal fragments (e.g., samples $10-13$ and $07-15$ ) are characterized by the lowest concentration of $\mathrm{SiO}_{2}$ (and by the greatest losses on ignition) compared with the other samples. The rocks of the cone edifice as sampled from the rockfall field (see Table 3, nos. 18-20) have chemical compositions that are consistent with andesite. It should be noted that this composition is also similar to the compositions of those ash samples where the admixture of hydrothermal material is insignificant.

\section{The Morphology of Ash Particles and the Composition of Volcanic Glass}

Three types of fragments were identified in ash samples for the larger explosive events of 2013-2016 and in the rockfall of July 2015 based on the morphology and structural features. Dense angular particles devoid of pores (Fig. 6a) dominate all of the samples. They contain 30 to $60 \%$ of all of the plagioclase and pyroxene microlites with occasional rounded disaggregations of quartz. Porous fragments (see Figs. 6c and 6d) are also present in each of the samples studied here, but their amounts are insignificant, 1 to 3 grains among a few hundreds of fragments. As well as the denser fragments, the porous fragments contain pyroxene and plagioclase microlites. Several porous particles were found to also contain discrete rounded grains of $\mathrm{FO}_{72-84}$ olivine in a thin reaction rim composed of orthopyroxene and clinopyroxene $(\mathrm{Mg} \#=$ 72-80). 
Table 1. Ash samples of Zhupanovsky Volcano, 2013-2016

\begin{tabular}{|c|c|c|c|c|c|}
\hline $\begin{array}{l}\text { Sample no. / } \\
\text { ashfall time }\end{array}$ & $\begin{array}{c}\text { Coordinates } \\
\text { of sampling sites, } \\
(\mathrm{N}, \mathrm{E})\end{array}$ & $\begin{array}{l}\text { Location of sampling sites } \\
\text { and distance to eruption center }\end{array}$ & $\begin{array}{c}\text { Ash mass, } \\
\mathrm{g} / \mathrm{m}^{2}\end{array}$ & $\begin{array}{l}\text { Sampling } \\
\text { date }\end{array}$ & Collector \\
\hline $\begin{array}{l}\mathbf{1 0 - 1 3}, \\
\text { October 24, } 2013\end{array}$ & $\begin{array}{l}53^{\circ} 33^{\prime} 32^{\prime \prime} \\
159^{\circ} 05^{\prime} 42^{\prime \prime}\end{array}$ & $\begin{array}{l}\text { Slopes of Second Cone, } \\
\text { alt. } 1187 \mathrm{~m}, \sim 3.5 \mathrm{~km} \text { northeast }\end{array}$ & 1100 & 26 Oct. 2016 & S.B. Samoilenko \\
\hline $\begin{array}{l}\mathbf{0 6} / \mathbf{1 - 1 4} \\
\text { June } 7-10,2014\end{array}$ & $\begin{array}{l}53^{\circ} 35^{\prime} 56^{\prime \prime}, \\
159^{\circ} 12^{\prime} 31^{\prime \prime}\end{array}$ & $\begin{array}{l}\text { Slopes of Second Cone, } \\
\text { alt. } 1115 \mathrm{~m}, \sim 4 \mathrm{~km} \text { east }\end{array}$ & 1732 & 10 Jun 2014 & T.M. Manevich \\
\hline $\begin{array}{l}\mathbf{0 6} / \mathbf{2 - 1 4} \\
\text { June 7, } 2014\end{array}$ & $\begin{array}{l}53^{\circ} 34^{\prime} 20^{\prime \prime}, \\
159^{\circ} 44^{\prime} 53^{\prime \prime}\end{array}$ & $\sim 40 \mathrm{~km}$ east & 126 & 10 Jun 2014 & T.M. Manevich \\
\hline $\begin{array}{l}\mathbf{0 6} / \mathbf{3 - 1 4}, \\
\text { June 7, } 2014\end{array}$ & $\begin{array}{l}53^{\circ} 34^{\prime} 27^{\prime \prime}, \\
159^{\circ} 24^{\prime} 36^{\prime \prime}\end{array}$ & $\sim 20 \mathrm{~km}$ east & 276 & 10 Jun 2014 & T.M. Manevich \\
\hline $\begin{array}{l}\text { 12-14, } \\
\text { December 29, } 2014\end{array}$ & $\begin{array}{l}53^{\circ} 30^{\prime} 28^{\prime \prime} \\
158^{\circ} 45^{\prime} 28^{\prime \prime}\end{array}$ & $\begin{array}{l}\text { Hot Nalychevo springs, } \sim 23 \mathrm{~km} \\
\text { southwest }\end{array}$ & 75 & 22 Jan 2015 & A.Yu. Fedoseev \\
\hline $\begin{array}{l}\mathbf{0 1 - 1 5} \\
\text { January 16, } 2015\end{array}$ & $\begin{array}{l}53^{\circ} 30^{\prime} 28^{\prime \prime} \\
158^{\circ} 45^{\prime} 28^{\prime \prime}\end{array}$ & $\begin{array}{l}\text { Hot Nalychevo springs, } \sim 23 \mathrm{~km} \\
\text { southwest }\end{array}$ & 500 & 22 Jan 2015 & A.Yu. Fedoseev \\
\hline $\begin{array}{l}\mathbf{0 1} / \mathbf{1 - 1 5} \\
\text { January 16, } 2015\end{array}$ & $\begin{array}{l}53^{\circ} 34^{\prime} 31^{\prime \prime}, \\
158^{\circ} 56^{\prime} 19^{\prime \prime}\end{array}$ & $\begin{array}{l}\text { Hot Talovsky springs, } \sim 18 \mathrm{~km} \\
\text { southwest }\end{array}$ & 160 & 31 Jan 2015 & Ya.D. Murav’ev \\
\hline $\begin{array}{l}\mathbf{0 1} / \mathbf{2 - 1 5} \\
\text { January 16, } 2015\end{array}$ & $\begin{array}{l}53^{\circ} 14^{\prime} 24^{\prime \prime}, \\
159^{\circ} 06^{\prime} 30^{\prime \prime}\end{array}$ & $\begin{array}{l}\text { East base of Kozelsky Volcano, } \\
\sim 38 \mathrm{~km} \text { south }\end{array}$ & 11.2 & 29 Apr 2015 & T.M. Manevich \\
\hline $\begin{array}{l}\mathbf{0 2 - 1 5} \\
\text { February } 2015\end{array}$ & $\begin{array}{l}53^{\circ} 21^{\prime} 28^{\prime \prime} \\
158^{\circ} 55^{\prime} 53^{\prime \prime}\end{array}$ & $\begin{array}{l}\text { East base of Koryaksky Volcano, } \\
\sim 32 \mathrm{~km} \text { south }\end{array}$ & 18 & 13 My 2015 & T.M. Manevich \\
\hline $\begin{array}{l}\mathbf{0 3 - 1 5} \\
\text { March } 2015\end{array}$ & $\begin{array}{l}53^{\circ} 34^{\prime} 31^{\prime \prime}, \\
158^{\circ} 56^{\prime} 19^{\prime \prime}\end{array}$ & $\begin{array}{l}\text { Hot Talovsky springs, } \sim 18 \mathrm{~km} \\
\text { southwest }\end{array}$ & 50 & 31 Mar 2015 & Ya.D. Murav’ev \\
\hline $\begin{array}{l}\mathbf{0 7 - 1 5} \\
\text { July 12, } 2015\end{array}$ & $\begin{array}{l}53^{\circ} 10^{\prime} 24^{\prime \prime} \\
159^{\circ} 20^{\prime} 47^{\prime \prime}\end{array}$ & Cape Nalychevo, $\sim 48 \mathrm{~km}$ southeast & 40 & 15 Jul 2015 & S.M. Volosynin \\
\hline $\begin{array}{l}\mathbf{0 1 - 1 6} \\
\text { January 19-21, } \\
2016\end{array}$ & $\begin{array}{l}53^{\circ} 35^{\prime} 40^{\prime \prime} \\
159^{\circ} 05^{\prime} 51^{\prime \prime}\end{array}$ & $\begin{array}{l}\text { Slopes of Priemysh Cone, } \\
\text { alt. } 1984 \mathrm{~m}, \sim 1 \mathrm{~km} \text { southwest }\end{array}$ & 3737 & 23 Jan 2016 & N.V. Gorbach \\
\hline $\begin{array}{l}\mathbf{0 1} / \mathbf{1 - 1 6} \\
\text { January 19-21, } \\
2016\end{array}$ & $\begin{array}{l}53^{\circ} 34^{\prime} 31^{\prime \prime}, \\
158^{\circ} 56^{\prime} 19^{\prime \prime}\end{array}$ & $\begin{array}{l}\text { Hot Talovsky springs, } \sim 18 \mathrm{~km} \\
\text { southwest }\end{array}$ & 106 & 22 Jan 2016 & A.Yu. Fedoseev \\
\hline $\begin{array}{l}\mathbf{0 1} / \mathbf{2 - 1 6}, \\
\text { January 21, } 2016\end{array}$ & $\begin{array}{l}53^{\circ} 27^{\prime} 14^{\prime \prime} \\
158^{\circ} 36^{\prime} 52^{\prime \prime}\end{array}$ & Pinachevo Pass, $\sim 36 \mathrm{~km}$ southwest & 6 & 10 Feb 2016 & A.G. Manevich \\
\hline $\begin{array}{l}\mathbf{0 2 - 1 6} \\
\text { February 6, } 2016\end{array}$ & $\begin{array}{l}53^{\circ} 30^{\prime} 23^{\prime \prime} \\
159^{\circ} 48^{\prime} 18^{\prime \prime}\end{array}$ & Lake Kalagir, $\sim 46 \mathrm{~km}$ east & 35 & 06 Feb 2016 & A.Yu. Fedoseev \\
\hline $\begin{array}{l}\mathbf{0 2} / \mathbf{1 - 1 6}, \\
\text { February 10, } 2016\end{array}$ & $\begin{array}{l}53^{\circ} 24^{\prime} 473^{\prime \prime} \\
159^{\circ} 37^{\prime} 07^{\prime \prime}\end{array}$ & $\begin{array}{l}\text { Lower reaches of Vakhil R., } \\
\sim 40 \mathrm{~km} \text { southeast }\end{array}$ & 26 & 13 Feb 2016 & A.Yu. Fedoseev \\
\hline $\begin{array}{l}\mathbf{0 2} / \mathbf{2 - 1 6}, \\
\text { February 13, } 2016\end{array}$ & $\begin{array}{l}53^{\circ} 23^{\prime} 174^{\prime \prime} \\
59^{\circ} 36^{\prime} 487^{\prime \prime}\end{array}$ & $\begin{array}{l}\text { Lower reaches of Vakhil R., } \\
\sim 46 \mathrm{~km} \text { southeast }\end{array}$ & 102.4 & 13 Feb 2016 & A.Yu. Fedoseev \\
\hline $\begin{array}{l}\text { 03-16, } \\
\text { March 25, } 2016\end{array}$ & $\begin{array}{l}53^{\circ} 19^{\prime} 29^{\prime \prime}, \\
157^{\circ} 31^{\prime} 49^{\prime \prime}\end{array}$ & Village Malki, $\sim 110 \mathrm{~km}$ southwest & 7 & 25 Mar 2016 & T.M. Manevich \\
\hline $\begin{array}{l}\mathbf{1 1 - 1 6} \\
\text { November 20, 2016 }\end{array}$ & $\begin{array}{l}53^{\circ} 31^{\prime} 170^{\prime \prime} \\
159^{\circ} 46^{\prime} 657^{\prime \prime}\end{array}$ & Lake Kalagir, $\sim 46 \mathrm{~km}$ east & 31 & 15 Feb 2017 & A.Yu. Fedoseev \\
\hline
\end{tabular}


Table 2. The brief characteristics of ash samples of larger explosive events and the material from the collapse of the sector in the cone

\begin{tabular}{c|c|c|c}
\hline $\begin{array}{c}\text { Sample no./ } \\
\text { date of eruptive } \\
\text { event }\end{array}$ & $\begin{array}{c}\text { Grain-size composition } \\
\text { (particle size - } \\
\text { percentage) }\end{array}$ & Main components & Sample microphotograph \\
\hline
\end{tabular}

Ash samples

\begin{tabular}{|c|c|c|c|}
\hline $\begin{array}{l}\text { 10-13/ } \\
\text { October } 2013\end{array}$ & $\begin{array}{l}<0.063-14.5 \% \\
0.063-0.125-38 \% \\
0.125-0.250-36 \% \\
0.250-0.5-12 \%\end{array}$ & $\begin{array}{l}\text { Fragments of hydrothermally altered rocks } \\
\text { and secondary minerals } \sim 60-70 \% \text {; } \\
\text { dense fragments and fragments of minerals } \sim 20 \% \text {; } \\
\text { porous glassy fragments are rare }\end{array}$ & $200 \mu \mathrm{m}$ \\
\hline $\begin{array}{l}06-14 / \\
\text { June } 2014\end{array}$ & $\begin{array}{l}<0.063-4.4 \% \\
0.063-0.125-19.7 \% \\
0.125-0.250-39.5 \% \\
0.250-0.5-34.9 \% \\
0.5-1.0-1.5 \%\end{array}$ & $\begin{array}{l}\text { Fragments of hydrothermally altered rocks } \\
\text { and secondary minerals } \sim 60 \% \text {; } \\
\text { dense fragments and fragments of minerals } \sim 30 \% \text {; } \\
\text { porous glassy fragments are rare }\end{array}$ & \\
\hline $\begin{array}{l}\text { 12-14/ } \\
\text { December } 2014\end{array}$ & $\begin{array}{l}<0.063-18.4 \% \\
0.063-0.125-37.1 \% \\
0.125-0.250-44.5 \%\end{array}$ & $\begin{array}{l}\text { Fragments of hydrothermally altered rocks and } \\
\text { secondary minerals } \sim 70-80 \% \text {; there are quartz- } \\
\text { feldspar aggregates with sulfide segregations; } \\
\text { dense fragments and fragments of minerals } \sim 20 \% \text {; } \\
\text { porous glassy fragments are rare }\end{array}$ & \\
\hline $\begin{array}{l}01-15 / \\
\text { January } 2015\end{array}$ & $\begin{array}{l}<0.063-7 \% \\
0.063-0.125-23.8 \% \\
0.125-0.250-21.7 \% \\
0.250-0.5-46.8 \% \\
0.5-1.0-0.7 \%\end{array}$ & $\begin{array}{l}\text { Dense fragments and fragments of minerals } \\
\sim 80-85 \% \text {; } \\
\text { fragments of hydrothermally altered rocks } \\
\text { and secondary minerals } \leq 15 \% \text {; } \\
\text { porous glassy fragments are rare }\end{array}$ & \\
\hline $\begin{array}{l}07-15 / \\
\text { July } 2015\end{array}$ & $\begin{array}{l}\text { Dominated } \\
\text { by a dust-like fraction }\end{array}$ & $\begin{array}{l}\text { Fragments of hydrothermally altered rocks } \\
\text { and secondary minerals } \sim 60-70 \% \text {; } \\
\text { dense fragments and fragments of minerals } \\
\sim 25-30 \% \text {; porous glassy fragments are rare }\end{array}$ & \\
\hline
\end{tabular}


Table 2. (Contd.)

\begin{tabular}{|c|c|c|c|}
\hline $\begin{array}{c}\text { Sample no./ } \\
\text { date of eruptive } \\
\text { event }\end{array}$ & $\begin{array}{c}\text { Grain-size composition } \\
\text { (particle size - } \\
\text { percentage) }\end{array}$ & Main components & Sample microphotograph \\
\hline $\begin{array}{l}01-16 / \\
\text { January } 2016\end{array}$ & $\begin{array}{l}<0.063-4.25 \% \\
0.063-0.125-10.63 \% \\
0.125-0.250-11.7 \% \\
0.250-0.5-17.02 \% \\
0.5-1.0-31.91 \% \\
1.0-2.0-20 \% \\
>2.0-4.49 \%\end{array}$ & $\begin{array}{l}\text { Dense fragments and fragments of minerals } \\
\sim 60-65 \% \text {; } \\
\text { hydrothermally altered particles } \leq 30 \% \text {; } \\
\text { porous glassy fragments and pumiceous particles } \\
\sim 7 \% \text {; a single angular fragment of homogeneous } \\
\text { glass }\end{array}$ & \\
\hline $\begin{array}{l}02-16 / \\
\text { February } 2016\end{array}$ & $\begin{array}{l}<0.063-1 \% \\
0.063-0.125-7 \% \\
0.125-0.250-31 \% \\
0.250-0.5-61 \%\end{array}$ & $\begin{array}{l}\text { Dense fragments and fragments of minerals } \sim 70 \% \text {; } \\
\text { hydrothermally altered particles } \sim 20 \% \text {; } \\
\text { porous glassy fragments and pumiceous particles } \\
\sim 5-7 \%\end{array}$ & \\
\hline $\begin{array}{l}03-16 / \\
\text { March } 2016\end{array}$ & $\begin{array}{l}\text { Dominated } \\
\text { by a dust-like fraction }\end{array}$ & $\begin{array}{l}\text { Dense fragments and fragments of minerals } \sim 60-70 \% \text {; } \\
\text { hydrothermally altered particles } \leq 20 \% \text {; } \\
\text { porous glassy fragments and pumiceous particles } \\
\sim 5 \%\end{array}$ & \\
\hline
\end{tabular}

Material from the July 2015 collapse of a sector in the cone.

$7650 /$ unconsolidated collapsed material \begin{tabular}{l|l}
$<0.063-1.7 \%$ & Fragments of hydrothermally altered rocks $\sim 70-75 \% ;$
\end{tabular} $0.063-0.125-5.15 \%$ there are quartz-feldspar aggregates with sulfide $0.125-0.250-24.33 \%$ phenocrysts;

$0.250-0.5-20.1 \%$

$0.5-1.0-16.77 \%$

$1.0-2.0-31.79 \%$ dense fragments $\sim 20-25 \%$; porous glassy frag-

ments are rare
7651-1/

blocky collapsed material
The sample is fragmented manually and is dominated by fragments $0.125-0.250 \mathrm{~mm}$ across
Porous and dense angular fragments and occasional fragments of minerals

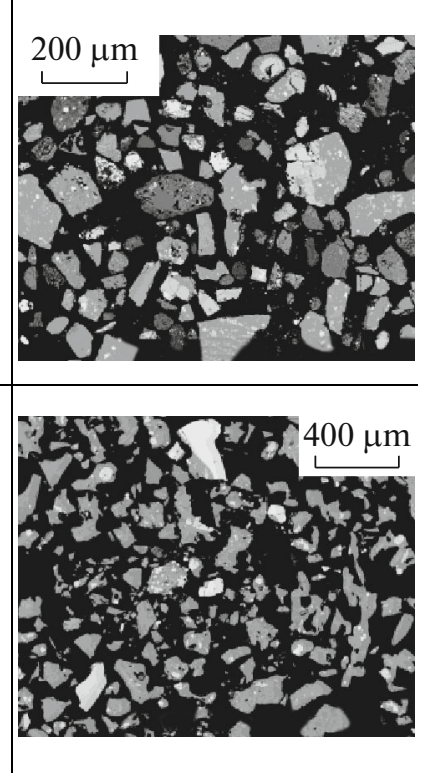



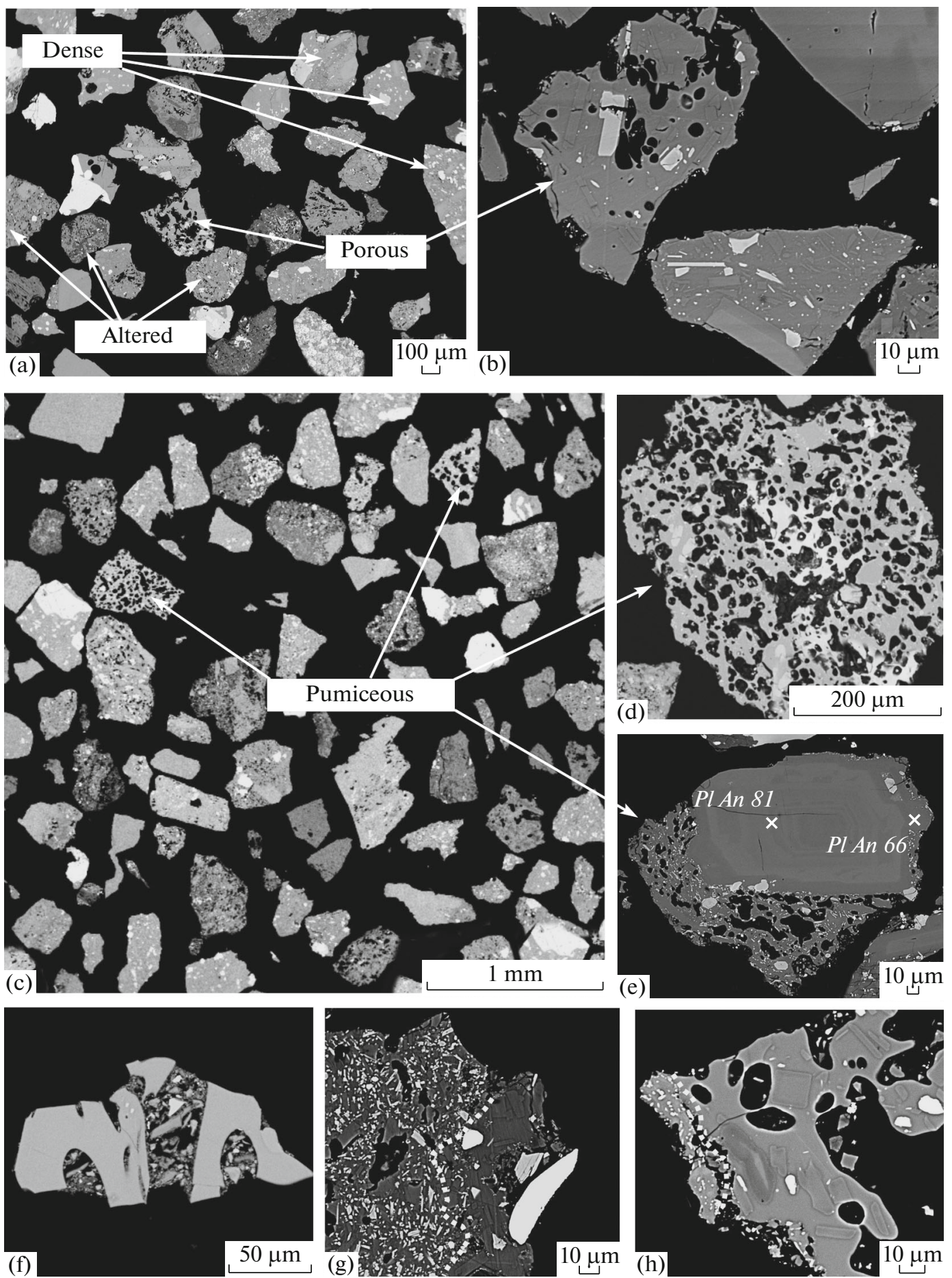

Fig. 4. The types of ash particles in the ejecta of Zhupanovsky Volcano. (a) dense, porous and hydrothermally altered particles in sample 01-16; (b) dense and porous fragments in sample 01-15; (c, d, e) pumiceous particles in sample 01-16; (f) a fragment of homogeneous glass (sample 01-16); (g, h)ash particles that combine patches with different structures (sample 02-16). The images in reflected electrons were obtained using a JEOL JXA 8200 electron microprobe and a SEM VEGA TESKAN scanning electron microscope.

Pumiceous particles were found in the ashes that were discharged in January, February, and March 2016 (see Fig. 6e). These particles, which are as large as $0.3-0.4 \mathrm{~mm}$ across, exhibit well-pronounced mor- phologic differences and possess a different character of recrystallization. In contrast to the first two types of fragment whose microlites are very definitely dominated by plagioclase (see Figs. 6b and 6d), the pumi- 


\begin{tabular}{|c|c|c|c|c|c|c|c|c|c|c|c|c|c|c|c|}
\hline ¿ి & $\frac{n}{i}$ & $\stackrel{f}{\sigma}$ & $\begin{array}{l}\tilde{C}_{0} \\
\stackrel{0}{0}\end{array}$ & $\begin{array}{l}\stackrel{0}{0} \\
\stackrel{6}{0}\end{array}$ & 1 & $\hat{\imath}$ & $\stackrel{m}{0}$ & $\underbrace{\infty}_{0}$ & in & 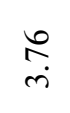 & $\stackrel{\mathcal{F}}{-}$ & Э0 & $\overrightarrow{0}$ & $\begin{array}{l}\hat{\infty} \\
\dot{\alpha} \\
\alpha\end{array}$ & 1 \\
\hline 2 & $\frac{\tilde{1}}{\hat{n}}$ & $\begin{array}{l}f \\
\text { di }\end{array}$ & 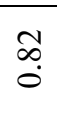 & 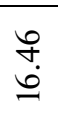 & 1 & 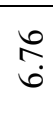 & $\stackrel{7}{0}$ & fo & $\underset{m}{\stackrel{m}{m}}$ & $\stackrel{\infty}{\stackrel{\infty}{r}}$ & $\stackrel{g}{g}$ & $\stackrel{0}{0}$ & $\overrightarrow{0}$ & $\begin{array}{l}\infty \\
\infty \\
\alpha\end{array}$ & 1 \\
\hline$\stackrel{\infty}{-}$ & $\frac{i}{\hat{n}}$ & $\begin{array}{l}n \\
i \\
i \\
n\end{array}$ & $\begin{array}{l}\stackrel{0}{\circ} \\
\stackrel{0}{0}\end{array}$ & $\begin{array}{l}\infty \\
\infty \\
ِ \\
ِ\end{array}$ & & 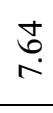 & $\stackrel{ \pm}{0}$ & $\stackrel{n}{\stackrel{n}{b}}$ & 8 & 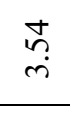 & $\vec{m}$ & $\Xi$ & $\stackrel{\text { on }}{0}$ & $\begin{array}{l}\infty \\
\infty \\
\alpha\end{array}$ & 1 \\
\hline$=$ & 危 & 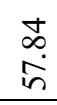 & $\stackrel{\text { ñ }}{\circ}$ & $\begin{array}{l}\stackrel{8}{+} \\
\stackrel{+}{+}\end{array}$ & 1 & 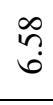 & $\stackrel{\circ}{\circ}$ & $\begin{array}{l}\stackrel{\circ}{\circ} \\
i\end{array}$ & $\underset{\sim}{\pi}$ & $\vec{\infty}$ & $\stackrel{9}{\exists}$ & $\frac{m}{0}$ & $\tilde{b}$ & $\begin{array}{l}n \\
\infty \\
\alpha \\
\alpha\end{array}$ & 1 \\
\hline$\underline{0}$ & 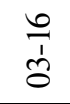 & $\stackrel{+}{b}$ & 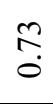 & 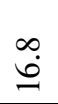 & $\stackrel{\sigma}{-}$ & $\underset{\sim}{\stackrel{+}{~}}$ & oे & f) & $\stackrel{\sim}{\text { N }}$ & 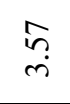 & $\widehat{\cong}$ & $\frac{n}{0}$ & $\stackrel{\text { I }}{i}$ & م. & $\hat{\jmath}$ \\
\hline$\stackrel{n}{ }$ & $\begin{array}{l}\frac{0}{1} \\
\dot{\delta}\end{array}$ & $\begin{array}{l}\infty \\
\stackrel{0}{0}\end{array}$ & $\hat{\imath}$ & $\stackrel{+}{\stackrel{\sigma}{\omega}}$ & $\alpha$ & $\stackrel{f}{f}$ & $\stackrel{7}{0}$ & $\begin{array}{l}\stackrel{\circ}{\circ} \\
\text { in }\end{array}$ & ¿े & $\stackrel{n}{m}$ & $\stackrel{\overbrace{}}{\rightarrow}$ & \pm & กิ & $\hat{\alpha}$ & $\stackrel{+}{\text { ț }}$ \\
\hline \pm & $\frac{0}{1}$ & $\frac{n}{6}$ & $\stackrel{n}{o}$ & $\stackrel{+}{\oplus}$ & 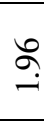 & $\stackrel{\infty}{\stackrel{\infty}{r}}$ & $\begin{array}{c}\infty \\
\infty \\
0\end{array}$ & 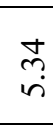 & $\stackrel{\infty}{\rightarrow}$ & $\stackrel{\vec{m}}{\dot{m}}$ & $\stackrel{2}{\exists}$ & $\frac{n}{0}$ & $\stackrel{\widehat{r}}{\tilde{m}}$ & $\begin{array}{l}\hat{g} \\
\stackrel{\alpha}{\alpha}\end{array}$ & $\underset{0}{+}$ \\
\hline$\cong$ & 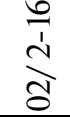 & : & $\stackrel{\hat{A}}{0}$ & $\hat{\sigma}$ & $\stackrel{\sim}{i}$ & $\stackrel{i}{n}$ & ठे. & के & $\stackrel{\widetilde{s}}{i}$ & $\stackrel{\tilde{m}}{\tilde{m}}$ & $\underset{I}{\simeq}$ & $\frac{n}{0}$ & $\stackrel{\infty}{\stackrel{\infty}{i}}$ & $\AA$ & స్ \\
\hline$\simeq$ & 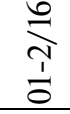 & 5 & $\stackrel{?}{\stackrel{0}{0}}$ & $\underset{\mathscr{m}}{m}$ & $\stackrel{M}{i}$ & $\stackrel{\infty}{\stackrel{\infty}{r}}$ & $\begin{array}{l}\infty \\
\stackrel{0}{\circ}\end{array}$ & $\stackrel{\sim}{+}$ & $\stackrel{\infty}{-}$ & $\stackrel{8}{\stackrel{8}{i}}$ & $\exists$ & $\stackrel{ \pm}{0}$ & $\begin{array}{l}\text { d } \\
\text { in }\end{array}$ & $\begin{array}{l}\text { న̆ } \\
\text { مू }\end{array}$ & : \\
\hline$=$ & $\stackrel{0}{0}_{0}^{0}$ & $\begin{array}{l}\infty \\
\stackrel{0}{0}\end{array}$ & $\stackrel{\infty}{\stackrel{0}{0}}$ & $\overbrace{}^{m}$ & $\approx$ & 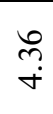 & $\stackrel{7}{0}$ & $\begin{array}{l}\text { के } \\
\text { in }\end{array}$ & $\stackrel{\underline{b}}{i}$ & $\vec{\sim}$ & $\fallingdotseq$ & $\stackrel{m}{0}$ & 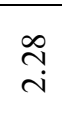 & $\begin{array}{l}3 \\
\alpha \\
\alpha\end{array}$ & శี \\
\hline 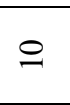 & $\frac{0}{1}$ & $\stackrel{0}{0}$ & $\begin{array}{l}\vec{\infty} \\
\stackrel{0}{0}\end{array}$ & 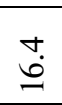 & $n$ & $\stackrel{\text { mे }}{\stackrel{1}{r}}$ & $\stackrel{7}{0}$ & $\begin{array}{l}\tilde{\omega} \\
\dot{n}\end{array}$ & $\bar{n}$ & $\underset{m}{\infty}$ & 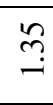 & $\stackrel{ \pm}{0}$ & 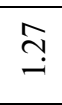 & $\begin{array}{l}n \\
m \\
\alpha\end{array}$ & : \\
\hline$a$ & $\frac{n}{1}$ & 官 & $\stackrel{R}{0}$ & $\stackrel{\leftrightarrow}{\stackrel{H}{a}}$ & & $\stackrel{\hat{N}}{\sim}$ & oे & कुे & $\stackrel{\overbrace{}}{i}$ & $\stackrel{m}{m}$ & $\exists$ & $\frac{ \pm}{0}$ & $\stackrel{\hat{\sigma}}{\hat{\sigma}}$ & $\begin{array}{l}\tilde{S} \\
\propto \alpha \\
\propto\end{array}$ & $\stackrel{9}{7}$ \\
\hline$\infty$ & $\frac{n}{d}$ & ñ & $\stackrel{0}{\circ}$ & $\underline{\varrho}$ & & $\stackrel{+}{m}$ & $\stackrel{\circ}{\circ}$ & $\stackrel{\sim}{i}$ & 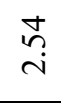 & $\stackrel{m}{m}$ & $\vec{I}$ & $\stackrel{m}{0}$ & ֶֻ. & $\hat{\alpha}$ & $\frac{\infty}{0}$ \\
\hline$r$ & \begin{tabular}{l}
$\frac{n}{1}$ \\
\multicolumn{1}{c}{}
\end{tabular} & $\therefore$ & $\stackrel{n}{\circ}$ & $\dot{\vec{\varphi}}$ & ț & $\stackrel{\infty}{m}$ & $\overline{0}$ & $\bar{n}$ & $\stackrel{\mathcal{F}}{i}$ & $\overrightarrow{\dot{m}}$ & $\stackrel{m}{\rightarrow}$ & $\stackrel{7}{0}$ & $\stackrel{a}{\stackrel{0}{0}}$ & aे & $\bar{o}_{0}^{0}$ \\
\hline 0 & $\stackrel{\frac{n}{1}}{\stackrel{1}{S}}$ & : & $\hat{\hat{o}}$ & $\underline{\underline{\omega}}$ & $\stackrel{\text { I }}{=}$ & $\stackrel{亏}{\stackrel{f}{f}}$ & $\stackrel{\circ}{\circ}$ & $\stackrel{\vec{b}}{\vec{n}}$ & $\stackrel{\mathfrak{a}}{i}$ & $\begin{array}{c}\stackrel{\infty}{\infty} \\
\dot{n}\end{array}$ & $\stackrel{\overbrace{}}{\cong}$ & $\stackrel{ \pm}{0}$ & $\stackrel{શ}{I}$ & $\begin{array}{l}\infty \\
\alpha \\
\alpha\end{array}$ & $\stackrel{\infty}{0}$ \\
\hline in & $\frac{n}{1}$ & $\overrightarrow{\tilde{b}}$ & 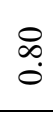 & $\begin{array}{l}\stackrel{+}{n} \\
\ddot{n}\end{array}$ & - & $\begin{array}{l}\stackrel{+}{\infty} \\
\dot{m}\end{array}$ & $\stackrel{\circ}{\circ}$ & $\begin{array}{l}\text { i্ } \\
i\end{array}$ & $\stackrel{\hat{i}}{\hat{i}}$ & $\stackrel{\partial}{\dot{r}}$ & $\stackrel{?}{=}$ & $\vec{m}$ & $\stackrel{\infty}{\rightarrow}$ & $\stackrel{\hat{\sigma}}{a}$ & $\overline{0}$ \\
\hline$\nabla$ & $\frac{n}{1}$ & $\overrightarrow{8}$ & $\stackrel{n}{\hat{o}}$ & $\stackrel{\text { I }}{ \pm}$ & $\begin{array}{l}\mathfrak{\sigma} \\
\text { in }\end{array}$ & $\stackrel{\sim}{m}$ & $\stackrel{\circ}{0}$ & $\stackrel{\leftrightarrow}{\stackrel{\leftrightarrow}{+}}$ & $\stackrel{⿱ 亠 凶 禸 ⺗}{\rightarrow}$ & $\stackrel{n}{i}$ & $\stackrel{\mathscr{\sigma}}{-}$ & $\frac{m}{0}$ & $\stackrel{m}{m}$ & ta. & $\tilde{n}$ \\
\hline$n$ & $\frac{\Xi}{\unlhd}$ & $\begin{array}{l}\text { : } \\
\text {. }\end{array}$ & $\stackrel{2}{\stackrel{0}{0}}$ & $\stackrel{R}{i}$ & $\approx$ & $\underset{\sim}{\tilde{f}}$ & $\frac{m}{0}$ & $\stackrel{\infty}{\stackrel{\infty}{+}}$ & $\underset{\sim}{\tilde{n}}$ & $\stackrel{\tilde{r}}{ }$ & $\stackrel{2}{=}$ & $\frac{J}{0}$ & $\begin{array}{l}\stackrel{0}{\infty} \\
\infty\end{array}$ & 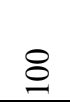 & $\stackrel{2}{0}$ \\
\hline$\sim$ & $\begin{array}{l}\frac{t}{b} \\
\stackrel{8}{8}\end{array}$ & $\stackrel{\circ}{6}$ & $\stackrel{\infty}{\stackrel{0}{0}}$ & $\stackrel{\nexists}{\underline{\theta}}$ & $\stackrel{\text { q}}{0}$ & $\stackrel{\widehat{N}}{i}$ & $\stackrel{7}{0}$ & $\stackrel{\infty}{\stackrel{\infty}{i}}$ & $\stackrel{t}{\stackrel{i}{j}}$ & $\stackrel{\tilde{r}}{\dot{r}}$ & $\stackrel{\infty}{\stackrel{\sim}{\sim}}$ & $\stackrel{t}{0}$ & $\stackrel{\circ}{\Im}$ & $\begin{array}{l}\dot{J} \\
\stackrel{0}{ }\end{array}$ & $\overrightarrow{\tilde{O}}$ \\
\hline- & $\frac{m}{\grave{b}}$ & $\stackrel{\vec{n}}{i}$ & 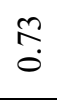 & 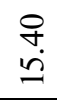 & $\bar{m}$ & $\stackrel{\infty}{\infty}$ & $\stackrel{m}{0}$ & ర్రి & $\stackrel{n}{+}$ & $\stackrel{\bar{m}}{\vec{m}}$ & $\cong$ & $\stackrel{n}{0}$ & $\stackrel{m}{+}$ & $\begin{array}{l}\hat{\jmath} \\
\infty \\
\alpha\end{array}$ & $\stackrel{\mathcal{F}}{-}$ \\
\hline $\begin{array}{l}\text { \# } \\
\text { 音 } \\
\text { o }\end{array}$ & 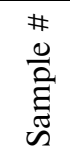 & in & $\stackrel{S}{\mathscr{O}}$ & 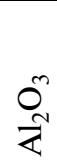 & $\underset{1}{0}$ & II & $\sum_{z}^{E}$ & 0 & $\Sigma$ & $\tilde{Z}^{\mathbb{Z}}$ & 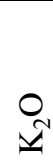 & $a$ & نـ & 吾 & $n$ \\
\hline
\end{tabular}




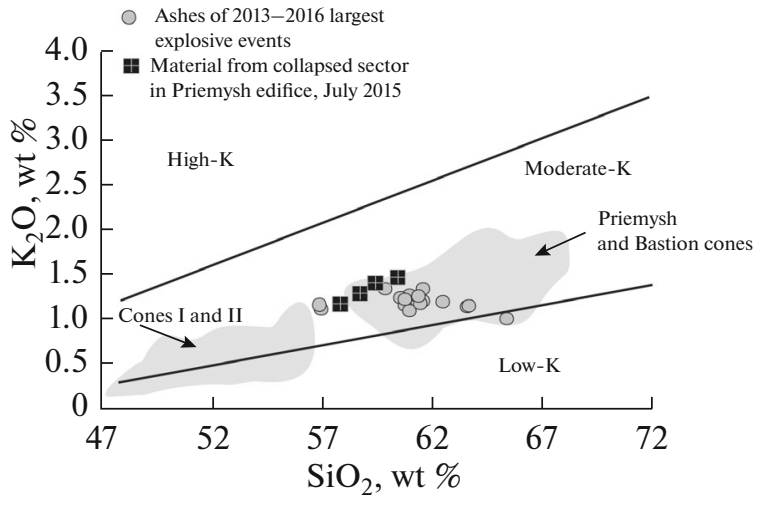

Fig. 5. The $\mathrm{SiO}_{2}-\mathrm{K}_{2} \mathrm{O}$ classification diagram for the 2013-2016 ejecta of Priemysh Cone. The compositional fields for Zhupanovsky Volcano are based on data from (Puzankov et al., 2016; Bazanova et al., 2009).

ceous fragments are saturated by minute pyroxene microlites (Fig. 6f). The relative amount of pumiceous particles is as high as ten per 200 grains. Some of these grains contain crystals of zonal high-calcium plagioclase (see Fig. 4e) and pyroxene. These same samples, which are due to the explosive events of the early 2016, were also found to contain occasional fragments of homogeneous volcanic glass (see Fig. 4f), as well as ash particles involving various combinations of recrystallized patches (see Figs. 4g and 4h).

Some representative compositions of volcanic glass from dense, porous, and pumiceous ash particles are listed in Table 4. Figure 7 shows the glass compositions in the $\mathrm{SiO}_{2}-\mathrm{K}_{2} \mathrm{O}, \mathrm{SiO}_{2}-\mathrm{CaO}, \mathrm{SiO}_{2}-\mathrm{MgO}$, and $\mathrm{SiO}_{2}-\mathrm{Cl}$ coordinates. For comparison purposes we also show the compositions of interstitial glasses in rocks of the cone edifice (sample 7651-1) sampled from the rockfall field. The compositions of most dense and porous fragments are identical and are consistent with rhyolite, while the pumiceous particles plot in the dacite field. All compositions follow the same trends, except for chlorine, whose behavior is different for particles with different concentrations of $\mathrm{SiO}_{2}$. The particles with the rhyolite composition of the glass (dense and porous fragments, as well as those from rocks in the edifice) follow the trend of a decreasing concentration of chlorine with a concomitant decreasing concentration of silica. The trend is less pronounced for the pumiceous particles.

The compositions of interstitial glasses sampled from the edifice that occur in all plots in the range of 74-78 wt \% silica are identical with the compositions of glasses from the dense and porous ash fragments.
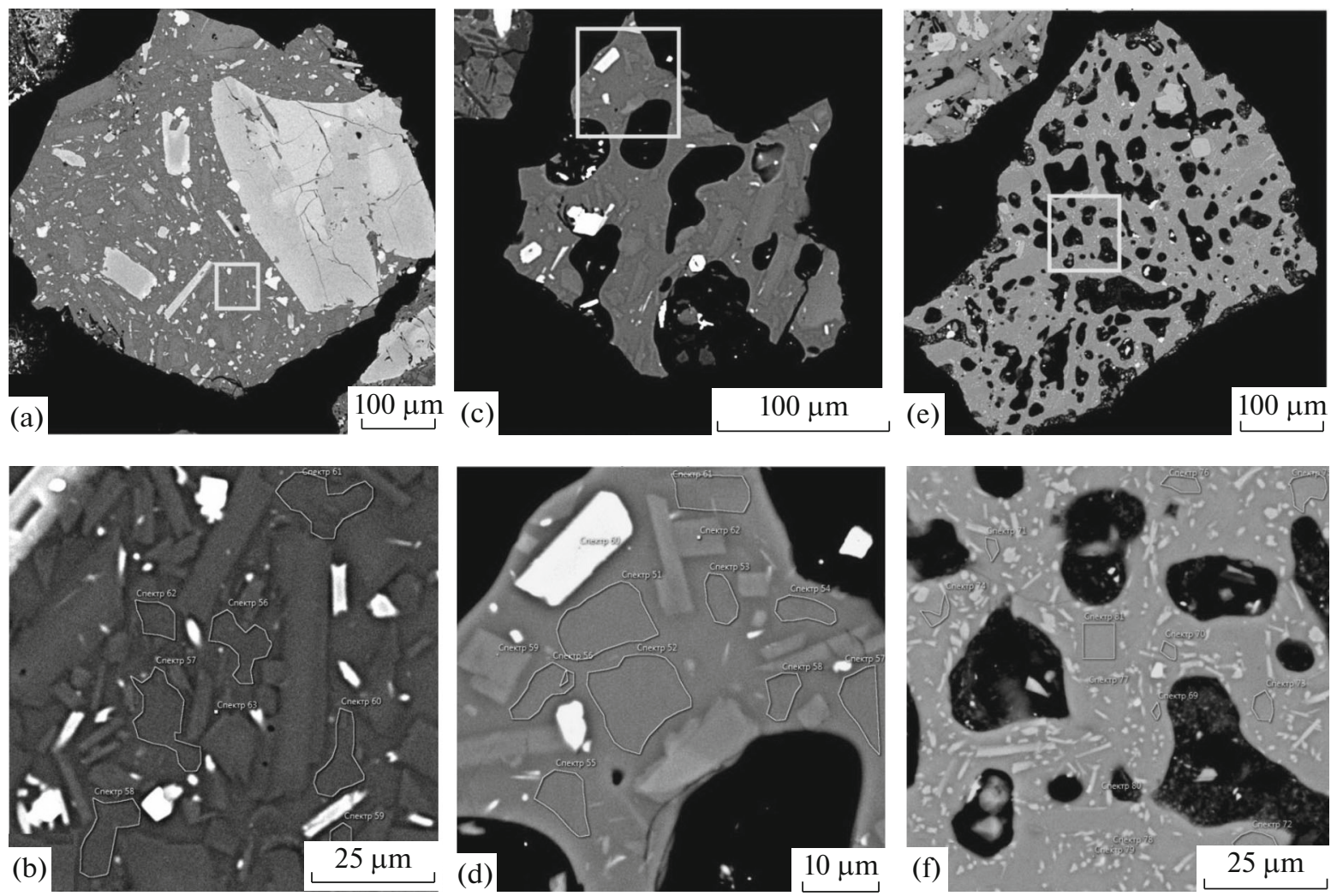

Fig. 6. The character of crystallization for various types of glassy fragments found in the ashes ejected in January 2016. (a, b) dense fragments, $(\mathrm{c}, \mathrm{d})$ porous fragments; $(\mathrm{e}, \mathrm{f})$ pumiceous particles. 
(a)

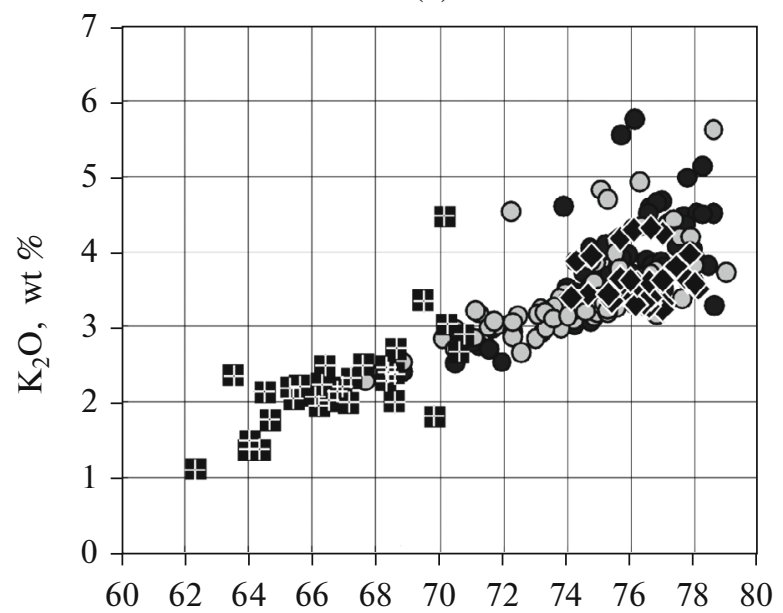

(c)

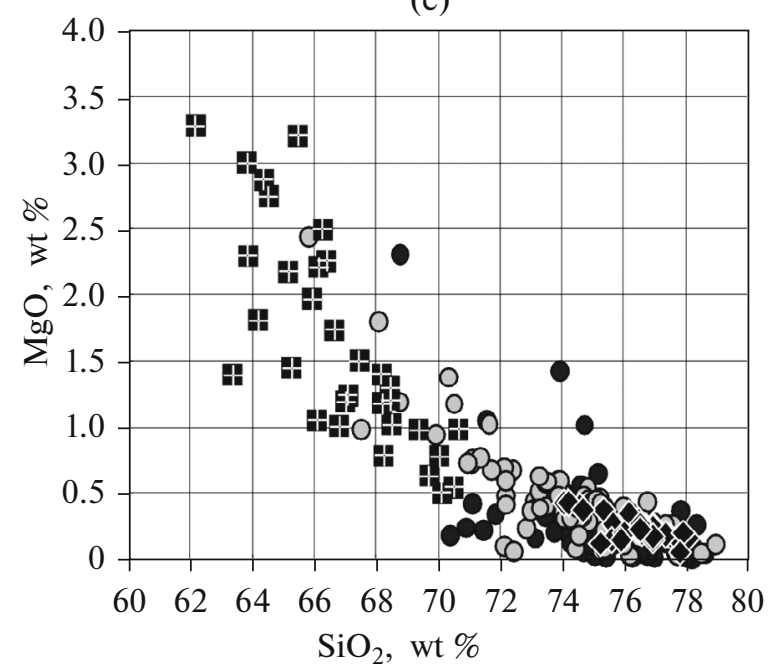

(b)

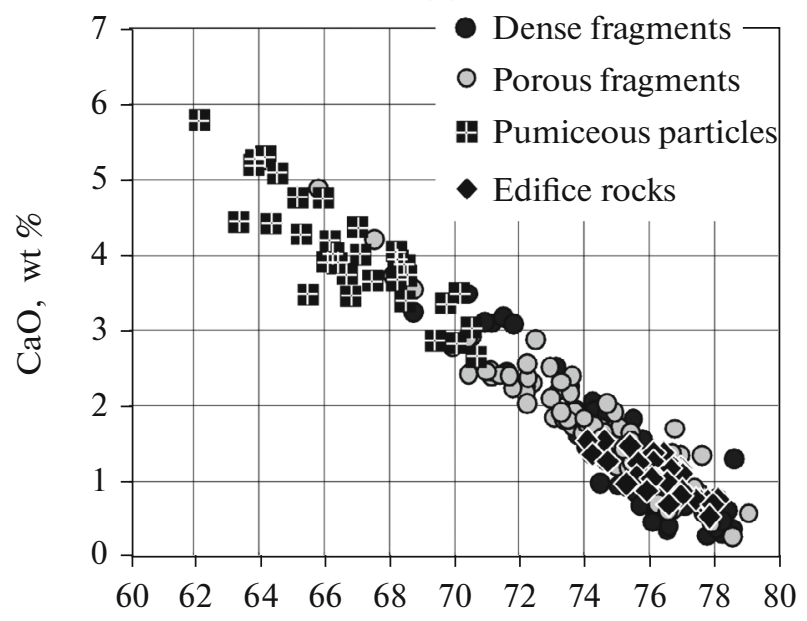

(d)

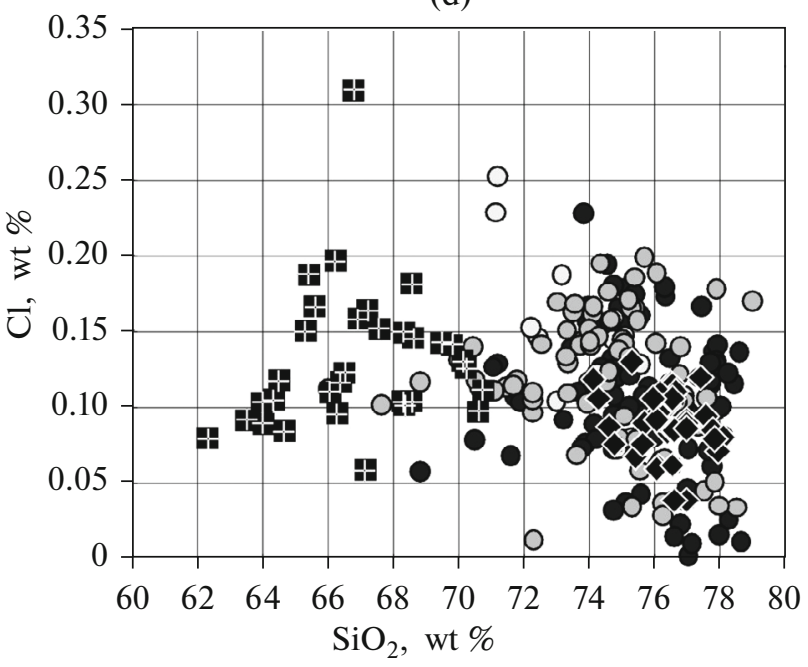

Fig. 7. The chemical composition of volcanic glass found in the 2013-2016 ejecta. The diagrams are based on glass compositions converted to dry basis.

\section{RESULTS AND DISCUSSION}

\section{The Identification of Juvenile Material}

The key issue for understanding the mechanism and character of an explosive eruption consists in determining the presence of juvenile magmatic material in the associated ejecta. The eruptions whose ejecta do not contain juvenile material are classified as phreatic. Such eruptions are caused by increasing pressure in the hydrothermal system of the volcano (Barberi et al., 1992, among others), while their ejecta (ash, ballistic fragments, and occasional pyroclastic flows) are composed of exclusively clastic material of the edifice and of products of hydrothermal activity. Phreatomagmatic eruptions are caused by emplacement and ascent of magma and its response to ground water and hydrothermal fluids. One diagnostic feature of such eruptions is the detection of fragments of juvenile material in the ejecta.
Juvenile material is most frequently identified by structural and petrologic analyses of ash particles. The juvenile particles mostly consist of fragments of thin glassy shards (Fig. 8a), which separated gas bubbles in the magma, of fragments with ragged edges (see Fig. $8 b$ ), or of very porous (see Fig. 8c) cinder or pumice fragments (Ponomareva et al., 2012; Liu et al., 2015). The particles of resurgent material or ejecta of phreatic activity are generally devoid of pores and have angular shapes (see Fig. 8d).

The determination of the chemical composition of volcanic glass by high-precision electron microprobe analysis is another effective tool to use for the identification of juvenile material. The best determinations of juvenile material were for a population of fragments with clear-cut glass compositions and definite morphology and structure (Pardo et al., 2014). However, if the glasses of the new eruption are compositionally close to the ejecta of the previous eruptions, then the 
Table 4. Representative analyses of volcanic glasses in the 2013-2016 ejecta of Zhupanovsky

\begin{tabular}{|c|c|c|c|c|c|c|c|c|c|c|c|c|c|c|c|}
\hline Sample\# & $\begin{array}{c}\text { Fragment } \\
\text { type }\end{array}$ & $\mathrm{SiO}_{2}$ & $\mathrm{TiO}_{2}$ & $\mathrm{Al}_{2} \mathrm{O}_{3}$ & $\mathrm{MnO}$ & $\mathrm{FeO}^{*}$ & $\mathrm{MgO}$ & $\mathrm{CaO}$ & $\mathrm{Na}_{2} \mathrm{O}$ & $\mathrm{K}_{2} \mathrm{O}$ & $\mathrm{P}_{2} \mathrm{O}_{5}$ & $\mathrm{~F}$ & $\mathrm{SO}_{3}$ & $\mathrm{Cl}$ & Total \\
\hline \multirow[t]{6}{*}{ 10-13 } & Dense & 73.33 & 0.62 & 13.43 & 0.05 & 2.57 & 0.39 & 1.90 & 4.18 & 3.18 & 0.13 & 0.07 & 0.01 & 0.13 & 100 \\
\hline & Dense & 74.98 & 0.44 & 13.55 & 0.05 & 1.38 & 0.11 & 1.76 & 4.20 & 3.26 & 0.06 & 0.09 & 0.00 & 0.13 & 100 \\
\hline & Dense & 77.48 & 0.57 & 11.47 & 0.08 & 1.45 & 0.11 & 0.75 & 3.55 & 4.05 & 0.11 & 0.20 & 0.00 & 0.17 & 100 \\
\hline & Porous & 73.03 & 0.67 & 13.69 & 0.11 & 2.57 & 0.36 & 2.07 & 4.07 & 3.15 & 0.06 & 0.02 & 0.02 & 0.17 & 100 \\
\hline & Porous & 74.26 & 0.63 & 12.65 & 0.09 & 2.87 & 0.41 & 1.63 & 3.92 & 3.23 & 0.09 & 0.05 & 0.02 & 0.15 & 100 \\
\hline & Porous & 76.06 & 0.56 & 12.64 & 0.06 & 1.69 & 0.10 & 1.14 & 3.76 & 3.59 & 0.09 & 0.13 & 0.00 & 0.19 & 100 \\
\hline \multirow[t]{6}{*}{ 06-14 } & Dense & 71.67 & 0.83 & 12.89 & 0.10 & 3.66 & 1.03 & 2.46 & 3.85 & 2.97 & 0.37 & 0.03 & 0.01 & 0.11 & 100 \\
\hline & Dense & 74.77 & 0.63 & 13.34 & 0.06 & 2.08 & 0.20 & 1.89 & 3.86 & 3.06 & 0.08 & 0.00 & 0.00 & 0.03 & 100 \\
\hline & Dense & 78.59 & 0.72 & 10.79 & 0.00 & 1.54 & 0.05 & 0.37 & 3.23 & 4.50 & 0.07 & 0.00 & 0.00 & 0.14 & 100 \\
\hline & Porous & 70.02 & 1.02 & 12.93 & 0.16 & 4.74 & 0.93 & 2.78 & 4.21 & 2.82 & 0.25 & 0.00 & 0.01 & 0.13 & 100 \\
\hline & Porous & 72.30 & 0.90 & 12.75 & \begin{tabular}{|l|}
0.09 \\
\end{tabular} & 3.63 & 0.58 & 2.01 & 4.30 & 3.07 & 0.20 & 0.05 & 0.00 & 0.11 & 100 \\
\hline & Porous & 75.31 & 0.64 & 12.50 & 0.11 & 2.09 & 0.47 & 1.47 & 3.97 & 3.18 & 0.09 & 0.00 & 0.00 & 0.17 & 100 \\
\hline \multirow[t]{6}{*}{$12-14$} & Dense & 74.56 & 0.64 & 12.42 & 0.10 & 2.21 & 0.32 & 1.70 & 4.31 & 3.27 & 0.19 & 0.12 & 0.02 & 0.15 & 100 \\
\hline & Dense & 71.22 & 0.63 & 15.16 & 0.03 & 2.13 & 0.41 & 3.10 & 4.25 & 2.73 & 0.19 & 0.01 & 0.01 & 0.13 & 100 \\
\hline & Dense & 77.71 & 0.14 & 11.97 & 0.05 & 0.91 & 0.13 & 0.57 & 3.84 & 4.48 & 0.02 & 0.08 & 0.04 & 0.07 & 100 \\
\hline & Porous & 71.14 & 0.86 & 13.47 & 0.17 & 3.37 & 0.72 & 2.44 & 4.13 & 3.20 & 0.19 & 0.05 & 0.03 & 0.23 & 100 \\
\hline & Porous & 75.12 & 0.55 & 12.49 & 0.02 & 2.40 & 0.31 & 1.51 & 4.06 & 3.27 & 0.06 & 0.06 & 0.01 & 0.14 & 100 \\
\hline & Porous & 74.22 & 0.54 & 13.07 & 0.07 & 2.48 & 0.30 & 1.77 & 4.17 & 3.10 & 0.11 & 0.03 & 0.03 & 0.12 & 100 \\
\hline \multirow[t]{6}{*}{ 01-15 } & Dense & 74.20 & 0.62 & 13.30 & 0.01 & 2.10 & 0.29 & 1.88 & 4.36 & 3.03 & 0.04 & 0.06 & 0.01 & 0.09 & 100 \\
\hline & Dense & 75.57 & 0.84 & 11.98 & 0.02 & 2.58 & 0.20 & 0.84 & 4.11 & 3.70 & 0.04 & 0.08 & 0.00 & 0.04 & 100 \\
\hline & Dense & 77.83 & 0.61 & 11.23 & 0.03 & 1.64 & 0.08 & 0.28 & 3.16 & 4.99 & 0.03 & 0.06 & 0.00 & 0.06 & 100 \\
\hline & Porous & 72.23 & 0.79 & 12.92 & 0.05 & 3.49 & 0.70 & 2.23 & 3.96 & 3.05 & 0.20 & 0.22 & 0.00 & 0.15 & 100 \\
\hline & Porous & 74.88 & 0.69 & 11.87 & 0.10 & 2.49 & 0.53 & 1.47 & 4.06 & 3.61 & 0.07 & 0.13 & 0.03 & 0.07 & 100 \\
\hline & Porous & 75.34 & 0.69 & 11.91 & \begin{tabular}{|l|}
0.07 \\
\end{tabular} & 2.69 & 0.37 & 1.15 & 3.99 & 3.54 & 0.09 & 0.05 & 0.03 & 0.08 & 100 \\
\hline \multirow[t]{5}{*}{ 07-15 } & Dense & 78.75 & 0.74 & 11.49 & 0.05 & 0.70 & 0.05 & 0.59 & 3.49 & 3.91 & 0.12 & 0.10 & 0.00 & 0.02 & 100 \\
\hline & Dense & 75.74 & 0.60 & 12.48 & 0.04 & 1.81 & 0.13 & 1.58 & 4.21 & 3.26 & 0.06 & 0.00 & 0.01 & 0.08 & 100 \\
\hline & Dense & 77.40 & 0.72 & 11.01 & 0.02 & 1.97 & 0.13 & 0.90 & 3.68 & 3.86 & 0.18 & 0.03 & 0.02 & 0.08 & 100 \\
\hline & Porous & 70.53 & 0.53 & 14.63 & 0.15 & 2.78 & 1.00 & 3.18 & 4.41 & 2.51 & 0.12 & 0.01 & 0.04 & 0.11 & 100 \\
\hline & Porous & 76.80 & 0.66 & 12.05 & 0.12 & 2.49 & 0.37 & 1.27 & 2.71 & 3.29 & 0.07 & 0.00 & 0.02 & 0.14 & 100 \\
\hline Sample \# & $\begin{array}{c}\text { Fragment } \\
\text { type }\end{array}$ & $\mathrm{SiO}_{2}$ & $\mathrm{TiO}_{2}$ & $\mathrm{Al}_{2} \mathrm{O}_{3}$ & $\mathrm{MnO}$ & $\mathrm{FeO}^{*}$ & $\mathrm{MgO}$ & $\mathrm{CaO}$ & $\mathrm{Na}_{2} \mathrm{O}$ & $\mathrm{K}_{2} \mathrm{O}$ & $\mathrm{P}_{2} \mathrm{O}_{5}$ & F & $\mathrm{SO}_{3}$ & $\mathrm{Cl}$ & Total \\
\hline \multirow[t]{8}{*}{ 01-16 } & Dense & 74.80 & 0.52 & 13.56 & 0.00 & 1.28 & 0.05 & 1.82 & 4.43 & 3.39 & 0.06 & 0.00 & 0.01 & 0.07 & 100 \\
\hline & Dense & 78.07 & 0.73 & 10.79 & 0.06 & 2.09 & 0.16 & 0.58 & 3.48 & 3.86 & 0.03 & 0.03 & 0.02 & 0.10 & 100 \\
\hline & Porous & 72.26 & 0.42 & 14.56 & 0.05 & 1.73 & 0.08 & 2.18 & 4.05 & 4.52 & 0.12 & 0.00 & 0.01 & 0.01 & 100 \\
\hline & Porous & 76.08 & 0.47 & 12.19 & 0.00 & 2.27 & 0.40 & 1.17 & 3.77 & 3.36 & 0.06 & 0.07 & 0.01 & 0.14 & 100 \\
\hline & Porous & 76.96 & 0.36 & 12.32 & 0.02 & 1.44 & 0.22 & 1.32 & 3.95 & 3.26 & 0.02 & 0.00 & 0.01 & 0.10 & 100 \\
\hline & Pumiceous & 64.27 & 0.80 & 16.15 & 0.15 & 4.40 & 1.80 & 5.30 & 5.20 & 1.37 & 0.18 & 0.13 & 0.14 & 0.10 & 100 \\
\hline & Pumiceous & 66.23 & 1.03 & 13.98 & 0.16 & 5.84 & 2.21 & 4.19 & 3.67 & 2.23 & 0.28 & 0.08 & 0.00 & 0.10 & 100 \\
\hline & Pumiceous & 69.79 & 1.06 & 13.80 & 0.07 & 4.63 & 0.63 & 3.35 & 4.43 & 1.81 & 0.28 & 0.00 & 0.02 & 0.14 & 100 \\
\hline \multirow[t]{5}{*}{$02-16$} & Dense & 75.84 & 0.41 & 12.26 & 0.02 & 2.18 & 0.24 & 1.53 & 3.83 & 3.48 & 0.06 & 0.06 & 0.01 & 0.08 & 100 \\
\hline & Dense & 78.58 & 0.28 & 11.58 & 0.03 & 0.75 & 0.04 & 0.25 & 2.74 & 5.61 & 0.01 & 0.11 & 0.01 & 0.03 & 100 \\
\hline & Porous & 73.36 & 0.68 & 12.98 & 0.04 & 2.35 & 0.63 & 2.32 & 3.56 & 3.74 & 0.13 & 0.08 & 0.01 & 0.11 & 100 \\
\hline & Porous & 75.29 & 0.17 & 13.62 & 0.00 & 0.82 & 0.07 & 0.92 & 4.35 & 4.68 & 0.03 & 0.02 & 0.00 & 0.03 & 100 \\
\hline & Porous & 76.31 & 0.19 & 12.98 & 0.06 & 0.97 & 0.01 & 0.68 & 3.80 & 4.92 & 0.00 & 0.05 & 0.01 & 0.04 & 100 \\
\hline
\end{tabular}


Table 4. (Contd.)

\begin{tabular}{l|l|l|l|l|l|l|l|l|l|l|l|l|l|l|l}
\hline Sample \# & $\begin{array}{l}\text { Fragment } \\
\text { type }\end{array}$ & $\mathrm{SiO}_{2}$ & $\mathrm{TiO}_{2}$ & $\mathrm{Al}_{2} \mathrm{O}_{3}$ & $\mathrm{MnO}$ & $\mathrm{FeO}$ & $\mathrm{MgO}$ & $\mathrm{CaO}$ & $\mathrm{Na}_{2} \mathrm{O}$ & $\mathrm{K}_{2} \mathrm{O}$ & $\mathrm{P}_{2} \mathrm{O}_{5}$ & $\mathrm{~F}$ & $\mathrm{SO}_{3}$ & $\mathrm{Cl}$ & $\mathrm{Total}$ \\
\hline \multirow{5}{*03-16}{} & Pumiceous & 66.02 & 0.62 & 14.52 & 0.13 & 5.25 & 1.97 & 4.75 & 4.32 & 2.08 & 0.19 & 0.05 & 0.00 & 0.11 & 100 \\
& Pumiceous & 68.59 & 0.97 & 14.38 & 0.01 & 4.00 & 1.01 & 3.73 & 4.17 & 2.71 & 0.24 & 0.04 & 0.00 & 0.15 & 100 \\
& Pumiceous & 70.58 & 0.80 & 14.30 & 0.00 & 3.45 & 0.54 & 3.04 & 4.26 & 2.67 & 0.21 & 0.06 & 0.00 & 0.10 & 100 \\
& Dense & 75.18 & 0.51 & 13.74 & 0.06 & 0.73 & 0.03 & 1.64 & 4.04 & 4.01 & 0.01 & 0.00 & 0.01 & 0.04 & 100 \\
& Dense & 76.59 & 0.60 & 11.97 & 0.00 & 1.75 & 0.04 & 0.38 & 3.77 & 4.50 & 0.05 & 0.00 & 0.33 & 0.01 & 100 \\
& Dense & 78.28 & 0.62 & 11.59 & 0.04 & 0.88 & 0.00 & 0.30 & 3.07 & 5.13 & 0.06 & 0.00 & 0.01 & 0.02 & 100 \\
$\mathbf{7 6 5 1 - 1}$ & Porous & 74.76 & 0.55 & 13.07 & 0.00 & 1.84 & 0.32 & 2.01 & 3.32 & 3.88 & 0.11 & 0.07 & 0.00 & 0.08 & 100 \\
& Porous & 76.15 & 0.71 & 12.25 & 0.04 & 2.04 & 0.11 & 1.00 & 4.15 & 3.35 & 0.09 & 0.00 & 0.00 & 0.11 & 100 \\
& Porous & 77.91 & 0.70 & 11.44 & 0.00 & 0.88 & 0.05 & 0.44 & 3.93 & 4.20 & 0.09 & 0.18 & 0.00 & 0.18 & 100 \\
& Pumiceous & 67.54 & 0.97 & 14.03 & 0.07 & 5.30 & 1.50 & 3.65 & 4.07 & 2.49 & 0.21 & 0.01 & 0.00 & 0.15 & 100 \\
& Pumiceous & 70.11 & 0.89 & 13.55 & 0.09 & 4.18 & 0.78 & 2.83 & 2.63 & 4.47 & 0.26 & 0.06 & 0.03 & 0.13 & 100 \\
& Porous & 72.97 & 0.54 & 14.43 & 0.08 & 1.90 & 0.22 & 2.49 & 4.36 & 2.83 & 0.07 & 0.00 & 0.01 & 0.10 & 100 \\
& Porous & 74.63 & 0.53 & 12.66 & 0.09 & 2.21 & 0.40 & 1.53 & 4.24 & 3.44 & 0.10 & 0.07 & 0.01 & 0.09 & 100 \\
& Porous & 76.13 & 0.55 & 12.11 & 0.00 & 2.15 & 0.29 & 1.27 & 3.97 & 3.30 & 0.05 & 0.09 & 0.00 & 0.10 & 100 \\
& Porous & 77.00 & 0.53 & 11.83 & 0.01 & 1.92 & 0.25 & 1.11 & 3.77 & 3.31 & 0.07 & 0.08 & 0.02 & 0.09 & 100 \\
\hline
\end{tabular}

identification of juvenile material becomes a difficult problem. As an example, studies of the ashes discharged by the ongoing (2010-2016) eruption of Turrialba Volcano in Costa Rica showed that the composition of the ash particles were identical with the ejecta of the previous major eruption and the supply of fresh magma is debatable (de Moor et al., 2016; Alvarado et al., 2016).

Of the three types of fragments detected in the Zhupanovsky ejecta, it is only the pumiceous particles in the ashes discharged in January-March 2016 that are identified as being definitely juvenile. The volcanic glasses in these particles have a dacitic composition different from the previous glasses, while their morphologic features meet all the criteria for the determination of juvenile material.

The origin of the porous fragments with a rhyolitic composition of glass in each sample of the 2014-2015 eruptions does not lend itself to an unambiguous interpretation. The morphologic features of these particles

(a)

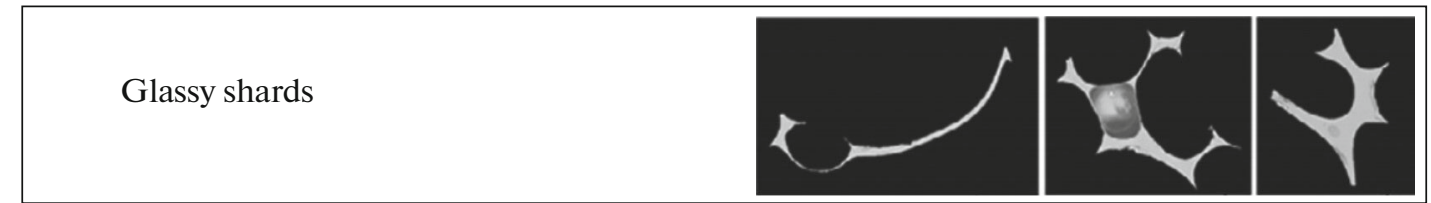

(b)

Porous

(c)

Microcrystalline porous

(d)

\begin{tabular}{|lllll}
\hline Dense & & \\
\hline
\end{tabular}

Fig. 8. Types of volcanic glass after (Liu et al., 2015). 
indicate a juvenile origin. At the same time, the chemical composition of their glass is indistinguishable from that of the glass in the dense fragments (see Fig. 7), while the lava sampled from the rockfall field and crushed manually exhibits a very similar aspect, in addition to a similar glass composition (see Table 2, sample 7651-1). However, the absence of secondary alteration in the porous fragments and occasional findings (in samples 01-16 and 03-16, January and March 2016) of splintered homogeneous rhyolitic volcanic glass (see Fig. 4f) are more likely to indicate a juvenile origin for the particles with a rhyolitic glass composition.

The origin of the dense fragments is ambiguous as well. They could have resulted either from fragmentation of edifice rocks (summit lavas) and/or could come from a magma portion cooling at a shallow depth. On the one hand, the fact of identical compositions for the volcanic glass from the denser fragments and for the interstitial glasses from lavas in the volcanic edifice provides evidence in favor of the hypothesis that the denser fragments might have resulted from fragmentation of the lavas that make the cone edifice. However, it cannot be ruled out that the denser particles might come from the upper degassed horizons of the magma column (or of the chamber) at shallow depths. Cashman and Hoblitt (2004) showed that the identification of juvenile material is complicated by the degassing and decompression-induced crystallization that accompany the slow ascent of magma and its residence at shallow depths. These processes lead to abundant crystallization of microlites and a high density of the ash particles. In addition, there is experimental evidence (Hammer and Rutherford, 2002) to show that a slow ascent of magma favors the formation of microlites with widely varying dimensions. It is these characteristics that describe most of the denser particles: the percentage of plagioclase microlites reaches $60 \%$, while their dimensions vary widely (see Figs. 6a and 6b).

According to Blundy and Cashman (2001), the presence of quartz and plagioclase in the groundmass and a higher (over $\sim 75 \mathrm{wt} \%$ ) concentration of $\mathrm{SiO}_{2}$ in volcanic glass indicates a shallow ( $\leq 50 \mathrm{MPa})$ crystallization environment. This environment is proper to shallow depths, less than $2 \mathrm{~km}$. If we attribute the quartz-bearing fragments with a high $\mathrm{SiO}_{2}$ glass composition to the 2013-2016 activity, then the crystallization must have actually occurred at the base of the volcanic edifice. As will be shown below, this inference is inconsistent with the dynamics of the eruption, thus favoring a resurgent origin of the denser particles.

\section{An Analysis of the Eruption Dynamics and Its Hypothetical Mechanism}

The most prominent features of the 2013-2016 Zhupanovsky eruption include the absence of signs that indicate extrusive or effusive activity during a long duration of explosive activity, the abundance of hydrothermal material in the ash, and the collapse of a sector in the edifice of the active cone. The small amount of the material that is reliably identified as juvenile indicates that the main trigger of the eruption was intensive degassing of emplaced, but still undischarged, magma. The analysis of such eruptions usually implies two possible scenarios, namely, magma emplacement at shallow depths or the intrusion of magma into deeper levels of the plumbing system (Moran et al., 2011).

We now compare the data on the 2013-2016 explosive activity (Fig. 9) and some features in ash composition from Table 2. The ejecta discharged during the first episode of this eruption in October 2013 contain the maximum amount of hydrothermally altered material. The ash sampled in an immediate vicinity of the eruption center (see Table 1, Table 2, sample 10-13) is mostly fine-grained, which is typical of phreatic eruptions (Barberi et al., 1992). It is most likely that the initial eruption episode was of a phreatic character, and can be viewed in retrospect as a precursor of the subsequent activity.

The ejecta of the ash discharges in the summer of 2014 were also dominated by hydrothermally altered material. This period of the eruption showed moderately vigorous explosive events, with the heights of the ejections rarely exceeding 4-6 km a.s.l. From November 2014, the vigor of individual ejections increases (see Fig. 9) and the ash discharged during this phase of activity began to be dominated by denser fragments. It was probably during this period that the deeper parts of the volcanic conduit became activated and the phreatic phase gave way to the phreatomagmatic phase.

The collapse of a sector in the edifice in July 2015 resulted in the release of the lithostatic load in the upper part of the plumbing system, with the conduit being truncated by a length of $500 \mathrm{~m}$. If magma resides at shallow depths, a rapid decompression generally leads to the eruption passing to the magmatic phase. The most conspicuous examples of this process are the 1956 Bezymyannyi eruption and the 1980 St. Helens eruption. The fact that the episode of activity followed the collapse of the sector on the Priemysh cone as late as 4 months after the collapse can be viewed as providing indirect evidence that no magma was present at shallow depths.

Comparatively rare, but powerful, explosive events occurred between late November 2015 and March 2016. The ashes of that period were found to contain a new type of juvenile particle with a dacitic composition of volcanic glass. It is likely that decompression in the upper part of the plumbing system of the volcano resulting from the collapse of a sector in the cone edifice in July 2015 involved material from deeper levels of the plumbing system in the eruption.

The explosive event of November 20, 2016 was separated from the preceding phase of activity by a span of 


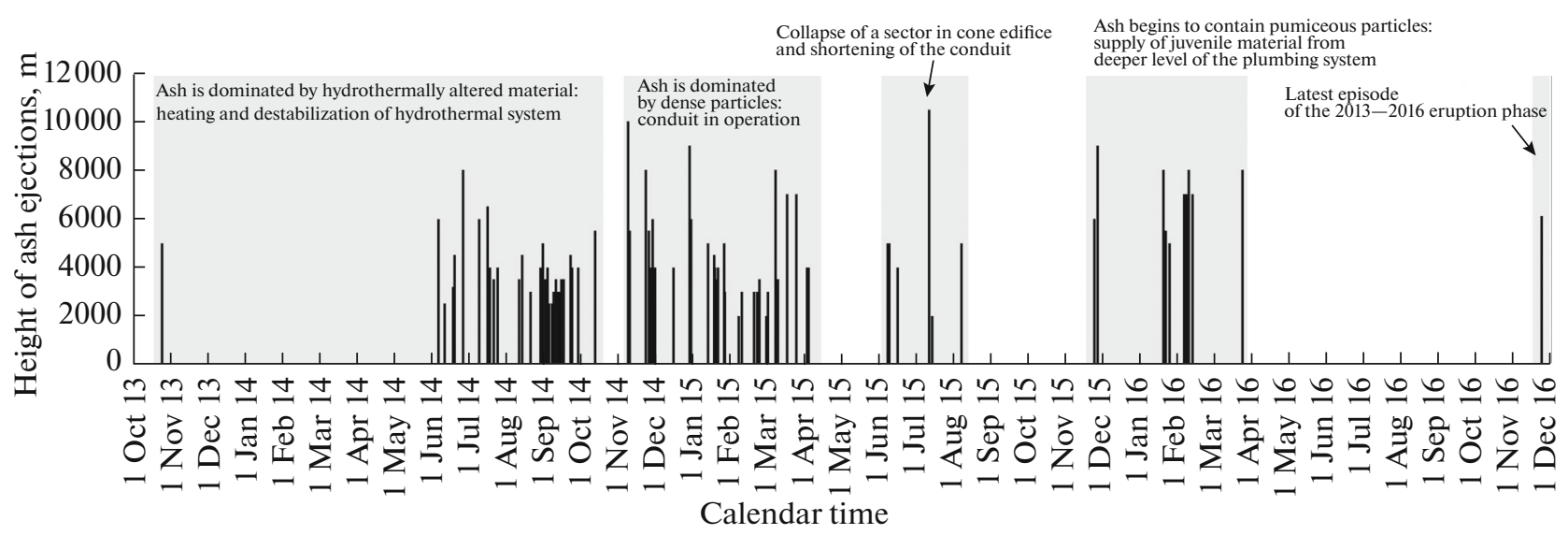

Fig. 9. The 2013-2016 explosive activity of the volcano and main ash characteristics. The data on the time and size of explosive events are given following the Kamchatka Volcanic Eruption Response Team http://www.kscnet.ru/ivs/kvert/ and the Laboratory of Research in Seismic and Volcanic Activity, Kamchatka Branch of the Federal Research Center, Unified Geophysical Survey, Russian Academy of Sciences http://www.emsd.ru/ ssl/monitoring/main.htm.

8 months. The event was isolated in time (see Fig. 9) and had no precursory seismicity; this makes this event similar to the first phase of activity in October 2013. The event was most likely phreatic and terminated the eruption phase of 2013-2016.

\section{CONCLUSIONS}

The 2013-2016 eruption activity of Zhupanovsky was predominantly phreatomagmatic, while no evidence of a magmatic activity, either effusive or extrusive, was recorded. The main components of the ashes are hydrothermally altered material and dense wellcrystallized particles whose origin is most likely due to the fragmentation of the rocks that compose the edifice and base of the volcano. The juvenile material, which consists of porous and pumiceous particles, is present in the ejecta in small amounts. The highest concentration of these particles (up to 5-7\%) was recorded in the ash discharged in January through March 2016. The ashes of that period of eruption were found to contain a new type of particle that is different from those discharged previously in morphology, structural features, and the composition of volcanic glass. It is thought that the decompression in the upper part of the plumbing system as a result of the collapse of a sector in the edifice of the active cone in July 2015 might involve material from deeper levels of the plumbing system in the eruption.

\section{ACKNOWLEDGMENTS}

We thank our colleagues at the Institute of Volcanology and Seismology FEB RAS Ya.D. Murav'ev, A.G. Manevich, and A.Yu. Fedoseev, the colleague at the Nalychevo Nature Park S.M. Volosynin for timely and careful sampling of ash, as well as Mario Turner for the aid he rendered us in the electron probe analy- sis at the Helmholtz Centre for Ocean Research (GEOMAR).

This work was supported in the framework of budget-sponsored research at the IV\&S FEB RAS and at the Vernadsky Institute of Geochemistry and Analytical Chemistry RAS, and in part through the Russian Foundation for Basic Research, project no. 15-0506440 and the Russian Science Foundation, project no. 16-07-10035.

\section{REFERENCES}

Alvarado, G., Mele, D., Dellino, P., et al., Are the ashes from the latest eruptions (2010-2016) at Turrialba volcano (Costa Rica) related to phreatic or phreatomagmatic events? J. of Volcanology and Geothermal Research, 2016, vol. 327, pp. 407-415.

Barberi, F., Bertagnini, A., Landi, P., and Principe, C., A review on phreatic eruptions and their precursors, $J$. of Volcanology and Geothermal Research, 1992, vol. 52, no. 4 , pp. $231-246$.

Bazanova, L.I., Dirksen, O.V., Kulish, R.V., and Kartasheva, E.V., The evolution of neotectonic volcanism at the Zhupanov Mountain Range, Kamchatka, in Vulkanizm i geodinamika (Volcanism and Geodynamics), Proc. IV All-Russia symp. on Volcanology and Paleovolcanology, Petropavlovsk-Kamchatskii: IViS DVO RAN, 2009, vol. 1, pp. 265-268.

Blundy, J. and Cashman, K., Ascent-driven crystallisation of dacite magmas at Mount St Helens, 1980-1986, Contributions to Mineralogy and Petrology, 2001, vol. 140, pp. 631-650.

Cashman, K.V. and Hoblitt, R.P., Magmatic precursors to the 18 May 1980 eruption of Mount St. Helens, USA, Geology, 2004, vol. 32, no. 2, pp. 141-144

de Moor, J.M., Aiuppa, A., Avard1, G., et al., Turmoil at Turrialba Volcano (Costa Rica): Degassing and eruptive processes inferred from high-frequency gas monitoring, J. of Geophysical Research: Solid Earth, 2016, vol. 121, pp. 5761-5775. doi 10.1002/2016JB013150 
Ermakov, V.A., Volynets, O.N., and Sapozhnikov, E.A., The Dzenzur-Zhupanovsky volcanic cluster, Byull. Vulkanol. St., 1973, no. 49, pp. 36-41.

Girina, O.A., Manevich, A.G., Mel'nikov, D.V., et al., The explosive activity of Zhupanovsky Volcano in 2016, in Vulkanizm i svyazannye s nim protsessy (Volcanism and Associated Processes), Proc. XIX regional conf. devoted to Volcanologist's Day, March 29-30, 2016, Petropavlovsk-Kamchatskii: IViS DVO RAN, 2016, pp. 24-34.

Gorbach, N.V., Samoilenko, S.B., Plechova, A.A., and Mel'nikov, D.V., The landslide on Zhupanovsky Volcano, Kamchatka in July 2015: First data and observations, Vestnik KRAUNTs, Nauki o Zemle, 2015, no. 3, issue 27 , pp. 5-11.

Gorshkov, A.P. and Slezin, Yu.B., The thermal power and the state of Zhupanovsky Volcano in 1970, Byull. Vulkanol. St., 1972, no. 48, pp. 29-32.

Hammer, J.E. and Rutherford, M.J., An experimental study of the kinetics of decompression-induced crystallization in silicic melt, J. of Geophysical Research, 2002, vol. 107, pp. 1-24.

Litvinov, A.F. and Burmakov, Yu.A., The geological structure and Quaternary volcanism of the Zhupanov mountain range, eastern Kamchatka, Vulkanol. Seismol., 1993, no. 2, pp. 16-26.

Liu, E.J., Cashman, K.V., and Rust, A.C., Optimising shape analysis to quantify volcanic ash morphology, GeoRes J., 2015, no. 8, pp. 14-30.

Manevich, T.M., Gorbach, N.V., and Samoilenko, S.B., Quantitative characteristics and ash composition: The 2013-2015 eruption of Zhupanovsky Volcano, in Vulkanizm i svyazannye s nim protsessy (Volcanism and Associated Processes), Proc. XVIII regional conf. devoted to Volcanologist's Day, March 30-April 1, 2015, Petropavlovsk-Kamchatskii: IViS DVO RAN, 2016, pp. 83-86. http://www.ivs.kscnet.ru/ivs/conferences/documents/tezis_2015.pdf

Masurenkov, Yu.P., Florenskii, I.V., and Melekestsev, I.V., Zhupanovsky Volcano, in Deistvuyushchie vulkany Kamchatki (The Active Volcanoes of Kamchatka), in two vols., Moscow: Nauka, 1991, vol. 2, pp. 216-225.

Moran, S.C., Newhall, C., and Roman, D.C., Failed magmatic eruptions: Late-stage cessation of magma ascent, Bulletin of Volcanology, 2011, vol. 73, no. 2, pp. 115122.

Nakada, S., Motomura, Y., and Shimizu, H., Manner of magma ascent at Unzen Volcano (Japan), Geophysical Research Letters, 1995, vol. 22, pp. 567-570.

Pardo N., Cronina S. J., Németh, K., et al., Perils in distinguishing phreatic from phreatomagmatic ash; insights into the eruption mechanisms of the 6 August $2012 \mathrm{Mt}$. Tongariro eruption, New Zealand, J. of Volcanology and Geothermal Research, 2014, vol. 286, pp. 397-414.

Piip, B.I., On the summit of Mount Zhupanovsky and on a recent eruption of that volcano, Byull. Vulkanol. St., 1947, no. 11, pp. 14-21.
Plechova, A.A., Portnyagin, M.V., and Bazanova, L.I., The origin and evolution of primary magmas in frontal volcanoes of Kamchatka based on a study of magmatic inclusions in the olivine of Zhupanovsky Volcano, Geokhimiya, 2011, no. 8, pp. 787-812.

Ponomareva, V.V., Portnyagin, M.V., and Mel'nikov, D.V., The composition of tephra from recent eruptions of volcanoes in Kamchatka and at the Kuril Islands, Vestnik KRAUNTs, Nauki o Zemle, 2012, no. 2, pp. 7-21.

Portnyagin, M., Borisova, A.Y., Plechov, P., et al., Magma mixing during the 2010 Eyjafjallajökull eruption, American Geophysical Union, 2011, Fall Meeting, Abstract. V33D-2676.

Puzankov, M.Yu., Bazanova, L.I., Dirksen, O.V., et al., Hybrid lavas of Priemysh cone: The Zhupanovsky Volcanic Cluster, Kamchatka, in Materialy regional'noi nauchnoi konferentsii "Vulkanizm i svyazannye s nim protsessy” posvyashchennoi Dnyu Vulkanologa (Proc. regional conference Volcanism and Associated Processes Devoted to Volcanologist's Day), March 29-30, 2016, Petropavlovsk-Kamchatskii: IViS DVO RAN, 2016, pp. 139-145.

Samoilenko, S.B., Mel'nikov, D.V., Chirkov, S.A., and Manevich, T.M., The activity increase on Zhupanovsky Volcano in 2013-2014, Vestnik KRAUNTs, Nauki o Zemle, 2014, no. 1, issue 23, pp.21-26.

Sigmarsson, O., Vlastelic, I., Andreasen, R., et al., Remobilization of silicic intrusion by mafic magmas during the 2010 Eyjafjallajökull eruption, Solid Earth, 2011, vol. 2, pp. 271-281.

Senyukov, S.L., Nuzhdina, I.N., Droznina, S.Ya., et al., The 2000-2015 seismicity of the Zhupanovsky Volcano area, in Problemy kompleksnogo geofizicheskogo monitoringa Dal'nego Vostoka Rossii (Problems in the Multidisciplinary Geophysical Monitoring of the Russian Far East), Proc. conf., September 27-October 3, 2015, Petropavlovsk-Kamchatskii, Obninsk: GS RAN, 2015, pp. 106-110. http://www.emsd.ru/conf2015lib/pdf/ mon/Senyukovetc.pdf

Sirin, A.N., The state of some volcanoes in Kamchtka in the early 1957, Byull. Vulkanol. St., 1958, no. 27, pp. 16-24.

Suzuki, Y., Nagai, M., Maeno, F., et al., Precursory activity and evolution of the 2011 eruption of Shinmoe-dake in Kirishima Volcano-Insights from ash samples, Earth Planets Space, 2013, vol. 65, pp. 591-607.

Taddeucci, J., Pompilio, M., and Scarlato, P., Monitoring the explosive activity of the July-August 2001 eruption of Mt. Etna (Italy) by ash characterization, Geophysical Research Letters, 2002, vol. 29, no. 8, pp. 71-74.

Watanabe, K., Danhara, T., Watanabe, K., et al., Juvenile volcanic glass erupted before the appearance of the 1991 lava dome, Unzen volcano, Kyushu, Japan, J. of Volcanology and Geothermal Research, 1999, vol. 89, pp. 13-121.

Translated by A. Petrosyan 